\title{
Methods for Computing and Modifying the $L D V$ Factors of a Matrix
}

\author{
By Philip E. Gill, Walter Murray and Michael A. Saunders
}

\begin{abstract}
Methods are given for computing the $L D V$ factorization of a matrix $B$ and modifying the factorization when columns of $B$ are added or deleted. The methods may be viewed as a means for updating the orthogonal $(L Q)$ factorization of $B$ without the use of square roots. It is also shown how these techniques lead to two numerically stable methods for updating the Cholesky factorization of a matrix following the addition or subtraction, respectively, of a matrix of rank one. The first method turns out to be one given recently by Fletcher and Powell; the second method has not appeared before.
\end{abstract}

1. Introduction. Any $m \times n$ matrix $B$ of rank $m(m \leqslant n)$ has an $L Q$ factorization of the form $B=\left[\begin{array}{ll}L & 0\end{array}\right] Q$, where $L$ is a nonsingular lower-triangular matrix and $Q$ is orthogonal $\left(Q^{T} Q=Q Q^{T}=I\right)$. The columns of $L$ and the first $m$ rows of $Q$ are uniquely defined, apart from sign. Let $l_{i i}$ be the diagonal elements of $L$ and let a diagonal matrix $D=\operatorname{diag}\left(d_{1}, d_{2}, \ldots, d_{n}\right)$ be defined by

$$
d_{i}= \begin{cases}l_{i i}^{2}, & i=1,2, \ldots, m, \\ 1, & i=m+1, \ldots, n\end{cases}
$$

An $L D V$ factorization of $B$ may then be written in the form $B=[\hat{L} 0] D V$, where $\hat{L}$ and $V$ are defined in terms of $L, Q$ and $D$ by the equations

$$
[\hat{L} 0] D^{1 / 2}=[L 0], \quad D^{1 / 2} V=Q .
$$

The diagonals of $\hat{L}$ are unity and the rows of $V$ are orthogonal. The following relations are easily proved:

$$
\begin{aligned}
V V^{T} & =D^{-1}, \\
V^{T} D V & =I .
\end{aligned}
$$

Henceforth we shall use the notation $L$ for both $L$ and $\hat{L}$ above, since it will always be clear from the context whether or not $L$ has a unit diagonal.

In this paper we derive methods for computing the $L D V$ factorization of a matrix and methods for modifying the factorization when columns are added and deleted. The resulting methods are described in Sections 3 and 4 and may be applied immediately to the Simplex method for linear programming. The motive for working with $L D V$ factors

Received December 3, 1974.

AMS (MOS) subject classifications (1970). Primary 65F30; Secondary 15A06, 15 A39, 90C05, $90 \mathrm{C20}, 90 \mathrm{C} 30$. 
rather than $L Q$ factors is that square roots are eliminated and the amount of computation and storage is reduced.

In Section 5 we show how these results lead naturally to two methods for computing the Cholesky factors $\bar{L}$ and $\bar{D}$ of the matrix $\bar{L} \bar{D} \bar{L}^{T}=L D L^{T}+\sigma z z^{T}$ for some vector $z$ and scalar $\sigma$. The method for the case $\sigma>0$ turns out to be one given by Fletcher and Powell (1973), while the method for the case $\sigma<0$ has not appeared before.

The keynote to this work is the construction of $L Q$ factors for two elementary matrices of the form

$$
\left[\begin{array}{ll}
I & p \\
& 1
\end{array}\right] \text { and } I-q q^{T}
$$

for given vectors $p$ and $q$, where $\|q\|_{2}=1$. The special structure of these factors is given in the Appendix. Although the derivation of the recurrence relations involved is relatively complicated, we emphasize that the recurrence relations themselves are very simple.

1.1. Notation. We shall use the notation $\tilde{M}=\tilde{M}(p, \beta, \gamma)$ to denote a special lower-triangular matrix constructed from the vectors $p, \beta$ and $\gamma$ according to

$$
\widetilde{M}_{i j}= \begin{cases}0, & i<j, \\ \gamma_{i}, & i=j, \\ p_{i} \beta_{j}, & i>j .\end{cases}
$$

If the diagonal elements of $\widetilde{M}$ form the vector $e=(1,1, \ldots, 1)^{T}$ we shall write either $\widetilde{M}=\tilde{M}(p, \beta, e)$ or just $\tilde{M}=\tilde{M}(p, \beta)$.

The notation $\|v\|$ will always mean the 2-norm $\|v\|_{2}=\left(v^{T} v\right)^{1 / 2}$ of a vector $v$, and a diagonal matrix $D$ with diagonals $d_{i}(i=1,2, \ldots, n)$ will be written $D=$ $\operatorname{diag}\left(d_{1}, d_{2}, \ldots, d_{n}\right)$.

2. $L D V$ Factors. We have defined in Section 1 what will be called a proper $L D V$ factorization of a general rectangular matrix $B$. For later use the notion needs to be generalized in the following way. Suppose that $L$ is unit lower triangular, $D$ is a diagonal matrix with positive diagonal elements, and $V$ is a matrix such that

$$
B=[L 0] D V .
$$

If there exist nonsingular diagonal matrices $D_{1}$ and $D_{2}$ such that the matrix $Q=D_{1} V D_{2}$ is orthogonal (unitary), then we shall call (2) an $L D V$ factorization of $B$. (In other words we require that $V$ can be transformed into an orthogonal matrix by simple row and column scaling.)

We now define (2) to be a proper $L D V$ factorization in the event that

$$
D_{1}=D^{1 / 2} \quad \text { and } \quad D_{2}=I \text {, }
$$

in which case $Q=D^{1 / 2} V$ and the relations 


$$
V V^{T}=D^{-1} \text { and } V^{T} D V=I
$$

hold as stated in Section 1.

We shall be particularly interested in the case where some of the columns of $B$ are null. Matrices $B$ of this kind will always arise in such a context that they are expressible in a form $L D V$ such that, corresponding to each $B e_{j}$ which is null, $L e_{j}=e_{j}, V e_{j}=$ $e_{j}, e_{j}^{T} V_{j}=e_{j}^{T}$ and $D e_{j}=0$, where $e_{j}$ is the $j$ th column of the identity matrix. If $D^{+}=$ $\operatorname{diag}\left(d_{1}^{+}, d_{2}^{+}, \ldots, d_{n}^{+}\right)$is defined by

$$
d_{i}^{+}= \begin{cases}d_{i}, & d_{i}>0 \\ 1, & d_{i}=0\end{cases}
$$

then the relations $V V^{T}=\left(D^{+}\right)^{-1}$ and $V^{T} D^{+} V=I$ hold in place of (1). It should be emphasized that when several columns of $B$ are null, the $L D V$ factorization is far from unique; the particular form of the factorization $L D V$ described above will arise in a natural way in the algorithms we describe.

3. Computing the $L D V$ Factorization of an $m \times m$ Matrix $B$. Let $A$ be a matrix made up of $j$ columns of $B$ and $m-j$ columns of the zero matrix (initially we shall not specify any particular ordering of the columns of $A$ ), and assume that the $L D V$ factorization of $A$, denoted by $A=L D V$, is known. We shall describe a method for computing the $L D V$ factors of the matrix $\bar{A}$ obtained by replacing a zero column of $A$ by a new column $b$. This technique leads naturally to a method for computing the $L D V$ factorization of $B$ since, if $B_{0}$ denotes the zero matrix with factorization $B_{0}=$ $L_{0} D_{0} V_{0}$, where $L_{0}=I, D_{0}=0$ and $V_{0}=I$, the columns of $B$ can be added one by one to $B_{0}$.

From our remarks in Section 2, the diagonal matrix associated with the factorization $A=L D V$ has $m-j$ zero elements and $V$ has $m-j$ columns of the identity matrix. Let $p$ be the vector such that $L p=b$, and $p_{s}$ the first element of $p$ such that $p_{s} \neq 0$ and $d_{s}=0$. Define

$$
\bar{A}=A+b e_{s}^{T}
$$

(that is, the column $b$ is added into the sth position). The recurrence relations we shall derive are invalid if $p_{s}=0$. However, if $B$ is nonsingular it can be shown that there exists at least one $\left|p_{j}\right|>0$ (otherwise the new column is a linear combination of those that have already been processed). Using the $L D V$ factorization of $A$, we have

$$
\bar{A}=L D V+b e_{s}^{T}=L\left(D V+p e_{s}^{T}\right)
$$

By definition, the sth row of $V$ is $e_{s}$, giving

$$
\bar{A}=L\left(D+p e_{s}^{T}\right) V .
$$

From Theorem A2 we have that the $L D V$ factorization of $D+p e_{s}^{T}$ is of the form

$$
D+p e_{s}^{T}=\tilde{L} \widetilde{D} \widetilde{V}
$$

where 
(5)

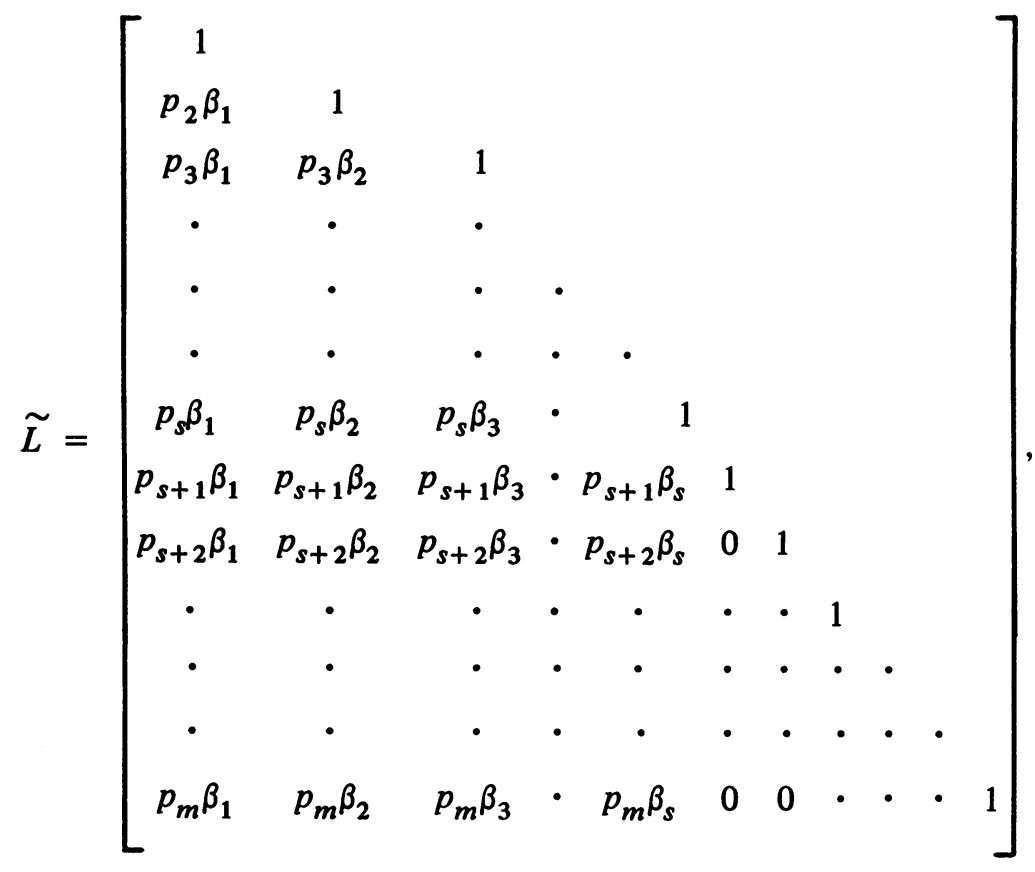

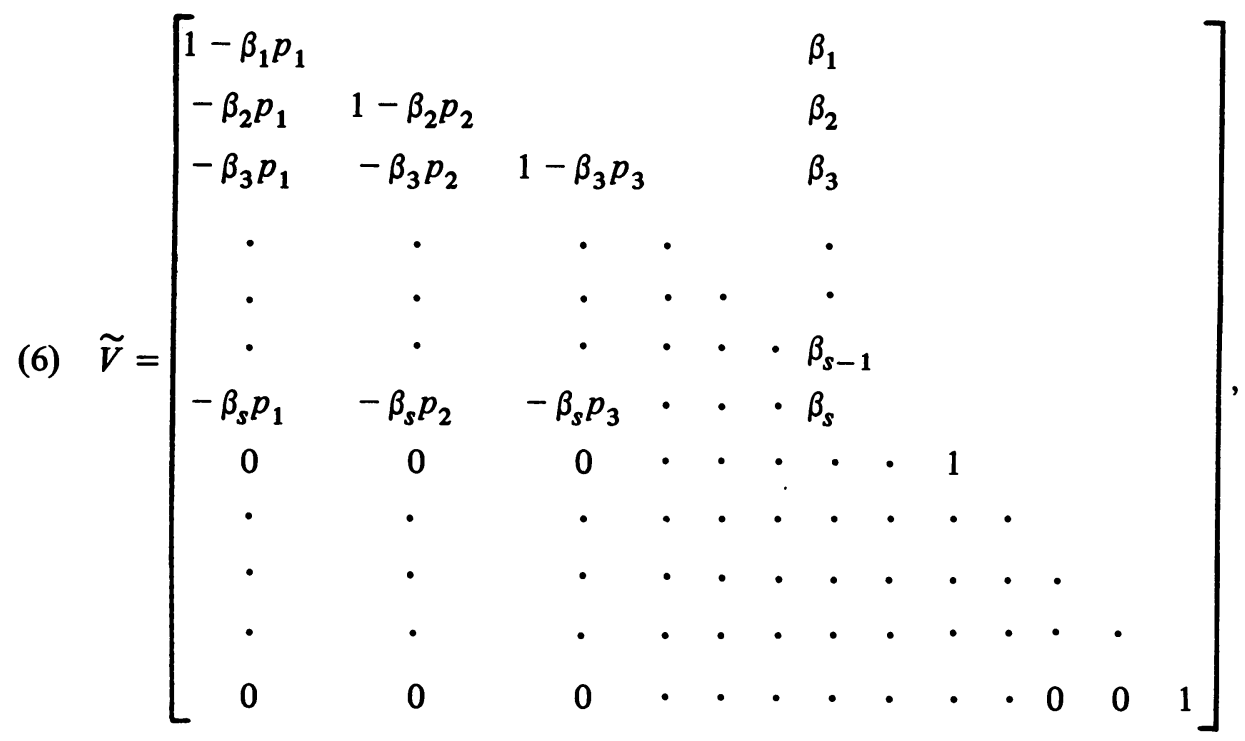

$$
\begin{aligned}
\widetilde{D} & =\operatorname{diag}\left(\tilde{d}_{1}, \tilde{d}_{2}, \ldots, \tilde{d}_{m}\right) \\
& =\operatorname{diag}\left(\hat{d}_{1}, \hat{d}_{2}, \ldots, \hat{d}_{s-1}, \hat{d}_{s}, d_{s+1}, \ldots, d_{m}\right)
\end{aligned}
$$

and the matrix $\left(\tilde{D}^{+}\right)^{1 / 2} \tilde{V}\left(D^{+}\right)^{-1 / 2}$ is orthogonal. The vectors $\hat{d}$ and $\beta$ are generated by the following recurrence relations:

(i) define $t_{0}=1$;

(ii) for $k=1,2, \ldots, s-1$ compute the following: if $d_{k}=0$, then set $\nu_{k}=0$, otherwise set $v_{k}=p_{k} / d_{k}, t_{k}=t_{k-1}+v_{k} p_{k}, \hat{d}_{k}=d_{k} t_{k} / t_{k-1}, \beta_{k}=v_{k} / t_{k}$; 
(iii) define $\hat{d}_{s}=p_{s}^{2} / t_{s-1}$ and $\beta_{s}=1 / p_{s}$.

Substituting (4) in (3) gives $\bar{A}=L \widetilde{L} \widetilde{D} \widetilde{V} V$. From the orthogonality of $\left(\widetilde{D}^{+}\right)^{1 / 2} \widetilde{V}\left(D^{+}\right)^{-1 / 2}$ we have

$$
\widetilde{V}\left(D^{+}\right)^{-1} \widetilde{V}^{T}=\left(\widetilde{D}^{+}\right)^{-1}
$$

Now

$$
(\widetilde{V} V)(\widetilde{V} V)^{T}=\widetilde{V} V V^{T} \widetilde{V}^{T}=\widetilde{V}\left(D^{+}\right)^{-1} \widetilde{V}^{T}=\left(\widetilde{D}^{+}\right)^{-1}, \text { from }(7)
$$

Consequently, if we write $\widetilde{V} V=\bar{V}, \widetilde{D}=\bar{D}$ and $L \widetilde{L}=\bar{L}$, then we have a factorization of $\bar{A}$ of the form required.

By adding each column of $B$ in turn and using the results just obtained we can generate a product form of the factorization (2). As the factorization proceeds, a new element of the diagonal matrix $D$ becomes nonzero and a new column of $L$ and column of $V$ are defined. Let $D_{j}, V_{j}$ and $L_{j}$ denote the matrices $\widetilde{D}, \widetilde{V}$ and $\widetilde{L}$ defined at (4) which are associated with the matrix made up of $j$ columns of $B$. Then we have

$$
B=L_{1} L_{2} \cdots L_{m} D_{m} V_{m} \cdots V_{2} V_{1},
$$

or $B=L D V$, if we write $L=L_{1} L_{2} \cdots L_{m}, V=V_{m} \cdots V_{2} V_{1}$ and $D=D_{m}$.

The important feature of the matrices $L_{j}$ and $V_{j}$ is that they both can be constructed from the pair of vectors $p$ and $\beta$. We shall show in Section 4.1 how their special form can be exploited to obtain the solution of equations of the form $L_{j} y=z$ and products of the form $y=V_{i} z$.

3.1. Stability and Sparseness Considerations. The general algorithm just given could be numerically unstable if the columns of $B$ were added in random order. Just as with $L U$ factorization, some "pivoting" strategy is required to ensure that the new column at each stage has a sufficiently large pivot element $\left(p_{s}\right.$ above). A preliminary ordering of the rows and columns of $B$ would reduce the amount of column interchanging required. In the context of linear programming, the preassigned pivot procedures of Hellerman and Rarick $(1971,1972)$ would be useful.

In general, the purpose of preassigned pivot procedures is to rearrange the rows and columns of an arbitrarily sparse matrix before the factorization commences in order to reduce the subsequent storage requirements. In mathematical terms we seek permutation matrices $P_{1}$ and $P_{2}$ such that the fill-in during the solution of the equations $P_{1} B P_{2} y=P_{1} b$, is less than that during the solution of $B x=b$. The solution $x$ can be obtained from $y$ using $x=P_{2} y$. One useful rearrangement of $B$, in view of the factorization being considered, is to choose $P_{1}$ and $P_{2}$ such that $P_{1} B P_{2}$ is of the form

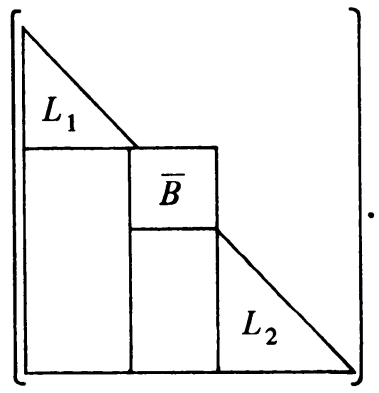


This matrix is lower triangular except for the matrix $\bar{B}$, defined as a bump. The lowertriangular matrices $L_{1}$ and $L_{2}$ are known as the forward triangle and backward triangle, respectively. Hellerman and Rarick (1971 and 1972) have given two algorithms for determining a further reordering of the matrix $\bar{B}$. These algorithms give a matrix $P_{1} B P_{2}$ as in (8) together with a matrix $\bar{B}$ which is itself lower triangular save for further bumps $B_{1}, B_{2}, \ldots, B_{j}$ (there may be any number), each of which is lower triangular save for columns of nonzero elements called spikes. For example, a bump $B_{j}$ could be of the form

$$
B_{j}=\left[\begin{array}{cccccc}
x & \cdot & \cdot & \cdot & \cdot & x \\
\cdot & x & \cdot & \cdot & \cdot & x \\
x & \cdot & x & x & \cdot & x \\
\cdot & \cdot & x & x & \cdot & \cdot \\
\cdot & x & x & \cdot & x & x \\
x & x & \cdot & x & x & \cdot
\end{array}\right]
$$

with $x$ denoting the nonzero elements. Our example has spikes in the fourth and last columns.

If we apply the $L D V$ factorization to a matrix which has been obtained by applying the Hellerman and Rarick scheme to $B$, then significant savings in fill-in are achieved. In this case, corresponding to a nonspike column, the $L_{j}$ is an elementary matrix and the $V_{j}$ is an identity matrix with its $j$ th diagonal element replaced by $1 / \beta_{j}$. The number of nontrivial $V_{j}$ 's is equal to the number of spike columns.

Rather than computing the $L D V$ factors of $B$ directly, there is an alternative strategy which maintains numerical stability and at the same time improves the sparsity of the factors. It is:

(1) compute a triangular factorization $B=L U$, using Gaussian elimination with column interchanges to preserve stability;

(2) use the above algorithm to compute an $L D V$ factorization of $U$. In this case it is natural to add the columns of $U$ in order from left to right.

The final result is a factorization of $B$ in the form

$$
B=L U=L L_{1} L_{2} \cdots L_{m} D_{m} V_{m} V_{m-1} \cdots V_{1} \text {. }
$$

Note that since $U$ is upper triangular the elements $p_{s+1}, \ldots, p_{m}$ are zero for each factor $L_{s}$.

This strategy has been implemented and tested on some medium-scale linear programs. The procedure $\mathrm{P}^{3}$ (Hellerman and Rarick (1971)) was used to specify an initial row and column ordering for $B$. (In practice only a few additional column interchanges are then required to ensure stability in the $L U$ factorization.) The recurrence relations defining the $L U$ factorization of $P_{1} B P_{2}$ imply that fill-in occurs only in the spike columns. For example, the $L U$ factorization of (9) is of the form 


$$
\left[\begin{array}{cccccc}
x & \cdot & \cdot & \cdot & \cdot & \cdot \\
\cdot & x & \cdot & \cdot & \cdot & \cdot \\
x & \cdot & x & \cdot & \cdot & \cdot \\
\cdot & \cdot & \cdot & x & \cdot & \cdot \\
\cdot & x & x & x & x & \cdot \\
x & x & \cdot & x & x & x
\end{array}\right]\left[\begin{array}{cccccc}
x & \cdot & \cdot & \cdot & \cdot & x \\
\cdot & x & \cdot & \cdot & \cdot & x \\
\cdot & \cdot & x & x & \cdot & x \\
\cdot & \cdot & \cdot & x & \cdot & x \\
\cdot & \cdot & \cdot & \cdot & x & x \\
\cdot & \cdot & \cdot & \cdot & \cdot & x
\end{array}\right] .
$$

The important facts are that

(a) the bulk of the $L U$ factorization is in $L$, and

(b) $U$ is almost strictly diagonal (except for the spikes).

In practice we find that there is virtually no further fill-in in the spike columns during the $L D V$ factorization of $U$. To summarize, this means that for a general sparse matrix $B$ (of the type encountered in $L P$ ) it is possible to compute an orthogonal factorization $B=L D V$ in product form, whose density is only slightly greater than that of the triangular factorization $B=L U$. This is a surprising result.

4. Adding and Deleting Columns of $B$. When combined, the two theorems in this section show how the $L D V$ factors of a nonsingular square matrix $B$ can be modified when one column of $B$ is replaced by a new column.

THEOREM 1 (ADDING A COLUMN). Let $B$ be an $m \times m$ nonsingular matrix and let the $m \times(m+1)$ matrix $[B \quad 0]$ have a proper $L D V$ factorization $[B \quad 0]=[L \quad 0] D V$, where $L$ is unit lower triangular, $D=\operatorname{diag}\left(d_{1}, d_{2}, \ldots, d_{m}, 1\right)$ and $D^{1 / 2} V$ is orthogonal. If a column $a_{s}$ is added to $B$ to give the matrix $\bar{B}$, then $\bar{B}$ has a proper LDV factorization $\bar{B}=\left[\begin{array}{ll}B & a_{s}\end{array}\right]=[\bar{L} \quad 0] \bar{D} \bar{V}$, with

$$
\bar{L}=L \widetilde{M}, \quad \bar{D}=\operatorname{diag}\left(d_{1}, d_{2}, \ldots, d_{m}, \alpha_{1}^{2}\right), \quad \bar{V}=\hat{V} V,
$$

where

$$
L p=a_{s}, \quad \widetilde{M}=\widetilde{M}(p, \beta), \quad \tilde{N}=\widetilde{M}(p, \beta)^{T}-\beta p^{T}, \quad \hat{V}=\left[\begin{array}{cc}
\widetilde{N} & \beta \\
-p^{T} & 1
\end{array}\right],
$$

and $\bar{D}^{1 / 2} \bar{V}$ is orthogonal. The quantities $\bar{d}_{j}, \beta_{j}$ and $\alpha_{1}^{2}$ are defined by the following recurrence relations:

$$
\begin{aligned}
& 10(\mathrm{i}) \text { define } t_{0}=1 \\
& \text { 10(ii) } \text { for } j=1,2, \ldots, \text { m set } \\
& t_{j}=t_{j-1}+p_{j}^{2} / d_{j}, \\
& d_{j}=d_{j} t_{j} / t_{j-1}, \\
& \beta_{j}=p_{j} /\left(d_{j} t_{j}\right) \\
& 10 \text { (iii) define } \alpha_{1}^{2}=1 / t_{m} .
\end{aligned}
$$

Proof. Adding the column to $B$ gives

$$
\bar{B}=\left[\begin{array}{ll}
B & 0
\end{array}\right]+a_{s} e_{m+1}^{T}=[L 0] D V+L p e_{m+1}^{T}=[L 0]\left(D V+\left[\begin{array}{l}
p \\
0
\end{array}\right] e_{m+1}^{T}\right)
$$


where $p$ is the solution of $L p=a_{s}$. Now $D^{1 / 2} V$ is orthogonal and the last column of [B 0 ] is zero; hence $D^{1 / 2} V$ is really of the form

$$
D^{1 / 2} V=\left[\begin{array}{ll}
Q & 0 \\
0 & 1
\end{array}\right]
$$

where $Q$ is the orthogonal matrix in the $L Q$ factorization of $B$ itself. Since $d_{m+1}^{1 / 2}=1$ this means that $e_{m+1}^{T} V=e_{m+1}^{T}$. Substituting into (11) gives

$$
\bar{B}=\left[\begin{array}{ll}
L & 0
\end{array}\right]\left(D+\left[\begin{array}{l}
p \\
0
\end{array}\right] e_{m+1}^{T}\right) V=\left[\begin{array}{ll}
L & 0
\end{array}\right] \hat{L} \hat{D} \hat{V} V \equiv[\bar{L} 0] \bar{D} \bar{V},
$$

where we are now using the corollary of Theorem A2 to write down an $L D V$ factorization of $D+\left[\begin{array}{l}p \\ 0\end{array}\right] e_{m+1}^{T}$. Using the notation of the corollary of Theorem A2, we have

$$
\left[\begin{array}{ll}
\bar{L} & 0
\end{array}\right]=\left[\begin{array}{ll}
L & 0
\end{array}\right] \hat{L}=\left[\begin{array}{ll}
L & 0
\end{array}\right]\left[\begin{array}{ll}
\tilde{M} & 0 \\
\beta^{T} & 1
\end{array}\right]=[L \tilde{M} 0]
$$

so that

$$
\begin{gathered}
\bar{L}=L \widetilde{M} ; \quad \bar{D}=\hat{D} ; \\
\bar{V}=\hat{V} V .
\end{gathered}
$$

The structure of $\hat{V}, \widetilde{M}$ and $\widetilde{N}$ and the recurrence relations (10) also follow from the corollary of Theorem A2. Finally we have

$$
\bar{D}^{1 / 2} \bar{V}=\hat{D}^{1 / 2} \hat{V} V=\left(\hat{D}^{1 / 2} \hat{V} D^{-1 / 2}\right)\left(D^{1 / 2} V\right),
$$

where both parenthesized quantities are orthogonal matrices. It follows that $\bar{D}^{1 / 2} \bar{V}$ is orthogonal and the theorem is proved.

THEOREM 2 (DELETING A COLUMN). Let $B$ be an $m \times(m+1)$ matrix with a proper $L D V$ factorization $B=[L 0] D V$, where $L$ is unit lower triangular, $D=$ $\operatorname{diag}\left(d_{1}, d_{2}, \ldots, d_{m}, \alpha_{1}^{2}\right)$ is positive definite and $D^{1 / 2} V$ is orthogonal. If $\bar{B}$ is the matrix remaining after the rth column $a_{r}$ is deleted from $B$, then $\bar{B}$ is nonsingular, and

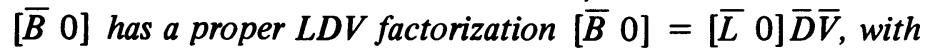

$$
\bar{L}=L \tilde{M}, \quad \bar{D}=\operatorname{diag}\left(\bar{d}_{1}, \bar{d}_{2}, \ldots, \bar{d}_{m}, 1\right), \quad \bar{V}=\hat{V} V \Pi,
$$

where

$$
\left[\begin{array}{c}
p \\
\alpha_{2}
\end{array}\right]=D V e_{r}, \quad \tilde{M}=\tilde{M}(p, \beta), \quad \hat{V}=\left[\begin{array}{cc}
\tilde{M}^{T} & \alpha_{2} \beta \\
p^{T} & \alpha_{2}
\end{array}\right], \quad \Pi=\text { a permutation matrix }
$$

and $\bar{D}^{1 / 2} \bar{V}$ is orthogonal. The quantities $\bar{d}_{j}$ and $\beta_{j}$ are defined by the following recurrence relations:

$$
\left.\begin{array}{l}
\text { 12(i) define } t_{m+1}=\alpha_{2}^{2} / \alpha_{1}^{2} ; \\
\text { 12(ii) for } j=m, m-1, \ldots, 1 \text { set } \\
t_{j}=t_{j+1}+p_{j}^{2} / d_{j}, \\
\begin{array}{l}
d_{j} \\
=
\end{array} d_{j} t_{j+1} / t_{j}, \\
\beta_{j}=-p_{j} /\left(d_{j} t_{j+1}\right) .
\end{array}\right\}
$$


Proof. If $\Pi$ is the permutation matrix which interchanges columns $r$ and $m+1$ of $B$, we have the identity

$$
\left[\begin{array}{ll}
\bar{B} & 0
\end{array}\right]=\left(B-a_{r} e_{r}^{T}\right) \Pi \text {. }
$$

Also, if we compute the $r$ th column of $D V$ as $\left[\begin{array}{c}p \\ \alpha_{2}\end{array}\right]=D V e_{r}$ we have

$$
a_{r}=B e_{r}=\left[\begin{array}{ll}
L & 0
\end{array}\right] D V e_{r}=\left[\begin{array}{ll}
L & 0
\end{array}\right]\left[\begin{array}{c}
p \\
\alpha_{2}
\end{array}\right]
$$

and

$$
e_{r}=V^{T}\left[\begin{array}{c}
p \\
\alpha_{2}
\end{array}\right]
$$

since $V^{T} D V=I$ from (1b). Substituting for $a_{r}$ and $e_{r}$ in (13) gives

$$
\left[\begin{array}{ll}
\bar{B} & 0
\end{array}\right]=\left[\begin{array}{ll}
L & 0
\end{array}\right]\left(D-\left[\begin{array}{c}
p \\
\alpha_{2}
\end{array}\right]\left[\begin{array}{ll}
p^{T} & \alpha_{2}
\end{array}\right]\right) V \Pi \text {. }
$$

Now from the definition of $p$ and $\alpha_{2}$ we have $\left\|D^{-1 / 2}\left[\begin{array}{c}p \\ \alpha_{2}\end{array}\right]\right\|=\left\|D^{1 / 2} V e_{r}\right\|=1$ since $D^{1 / 2} V$ is orthogonal. Hence the conditions of Theorem $\mathrm{A} 4$ are satisfied and we can write down an $L D V$ factorization of $D-\left[\begin{array}{c}p \\ \alpha_{2}\end{array}\right]\left[p^{T} \alpha_{2}\right]$ to give

$$
[\bar{B} 0]=[L \quad 0] \hat{L} \hat{D} \hat{V} V \Pi \equiv[\bar{L} 0] \bar{D} \bar{V}
$$

Using the notation of Theorem A4, we have

$$
\left[\begin{array}{ll}
\bar{L} & 0
\end{array}\right]=\left[\begin{array}{ll}
L & 0
\end{array}\right] \hat{L}=\left[\begin{array}{ll}
L & 0
\end{array}\right]\left[\begin{array}{ll}
\widetilde{M} & \\
\alpha_{2} p^{T} & 0
\end{array}\right]=\left[\begin{array}{ll}
L \widetilde{M} & 0
\end{array}\right]
$$

so that

$$
\begin{gathered}
\bar{L}=L \widetilde{M} ; \quad \bar{D}=\hat{D} ; \\
\bar{V}=\hat{V} V \Pi .
\end{gathered}
$$

The structure of $\hat{V}$ and $\widetilde{M}$ and the recurrence relations (12) also follow from Theorem A4. Finally we have

$$
\bar{D}^{1 / 2} \bar{V}=\hat{D}^{1 / 2} \hat{V} V \Pi=\left(\hat{D}^{1 / 2} \hat{V} D^{-1 / 2}\right)\left(D^{1 / 2} V\right)(\Pi),
$$

where all parenthesized quantities are orthogonal matrices. It follows that $\bar{D}^{1 / 2} \bar{V}$ is orthogonal and the theorem is proved.

Note also that the last row of $\bar{D}^{1 / 2} \bar{V}$ is

$$
\begin{aligned}
e_{m+1}^{T} \bar{D}^{1 / 2} \bar{V} & =e_{m+1}^{T} \hat{D}^{1 / 2} \hat{V} V \Pi=e_{m+1}^{T} \hat{V} V \Pi=\left[p^{T} \alpha_{2}\right] V \Pi \\
& =e_{r}^{T} V^{T} D V \Pi=e_{r}^{T} \Pi=e_{m+1}^{T},
\end{aligned}
$$

and hence $\bar{D}^{1 / 2} \bar{V}$ is of the form

$$
\bar{D}^{1 / 2} \bar{V}=\left[\begin{array}{ll}
\bar{Q} & \\
& 1
\end{array}\right]
$$


where $\bar{Q}$ is the orthogonal matrix in the $L Q$ factorization of $\bar{B}$.

Theorems 1 and 2 imply that when a column of $B$ is replaced by a new column we can extend the product form of Section 3 by adding new factors $L_{m+1}, V_{m+1}$, $L_{m+2}, V_{m+2}$ and updating $D_{m}$ to become $D_{m+2}$.

4.1. Use of the Special Matrices $\widetilde{M}$ and $\hat{V}$. The matrices $\widetilde{M}=\widetilde{M}(p, \beta)$ in Theorems 1 and 2 will be used to solve systems of the form

$$
\widetilde{M} y=z \text { or } \widetilde{M}^{T} y=z .
$$

Algorithms are given in Saunders (1972) which show that $y$ can be computed using two multiplication operations for each nonzero element in $p$. Similarly the matrices $\hat{V}$ in Theorems 1 and 2 will be used to compute products of the form

$$
y=\hat{V} z \quad \text { or } \quad y=\hat{V}^{T} z
$$

and it is easy to show that $y$ can again be computed using only two multiplies per nonzero element in $p$.

5. Modification of the Cholesky Factors. This section is concerned with the modification of the Cholesky factors of a symmetric positive-definite matrix $A$ after a rankone correction. In mathematical terms, the problem is to compute the Cholesky factors $\bar{L} \bar{D} \bar{L}^{T}$ such that

$$
\bar{L} \bar{D} \bar{L}^{T}=\bar{A}=A+\sigma z z^{T}=L D L^{T}+\sigma z z^{T} .
$$

It will be assumed throughout that the elements $d_{j}$ and $\bar{d}_{j}$ are positive, which implies that the matrices $\bar{A}$ and $A$ are positive definite. We shall scale the vector $z$ such that the modification (14) is either of the form

$$
\bar{L} \bar{D} \bar{L}^{T}=L D L^{T}+v v^{T}
$$

or

$$
\bar{L} \bar{D} \bar{L}^{T}=L D L^{T}-v v^{T} .
$$

Although this scaling requires an additional $n$ divisions and a square root, it minimizes the probability of overflow/underflow on the occasions when $\sigma$ is large and $\|z\|$ is small.

Since $A$ is positive definite, it can be written in the form $A=B B^{T}$, where $B$ is a nonsingular $m \times m$ matrix. If $B$ has the proper $L D V$ factorization $B=L D V$, then $L$ and $D$ are the Cholesky factors of $A$. The two methods given in 5.1 and 5.2 for performing the modifications (15) and (16), respectively, are based upon the theorems given in the appendix for modifying the $L D V$ factorization of $B$ without storing $V$.

5.1. $\bar{L} \bar{D} \bar{L}^{T}=L D L^{T}+v v^{T}$. We have the identity

$$
\bar{A}=L\left(D+p p^{T}\right) L^{T},
$$

where $p$ is the solution of the equations

$$
L p=v .
$$

We can now apply Lemma A3 to write down the $L D L^{T}$ factors of $D+p p^{T}$ as 


$$
D+p p^{T}=\widetilde{M} \widetilde{D} \widetilde{M}^{T},
$$

where $\widetilde{M}=\widetilde{M}(p, \beta)$ and $\widetilde{D}=\operatorname{diag}\left(\bar{d}_{1}, \ldots, \bar{d}_{m}\right)$ can be computed using the recurrence relations

$$
\left.\begin{array}{c}
\text { 19(i) define } t_{0}=1 ; \\
\text { 19(ii) for } j=1,2, \ldots, m \text { set } \\
t_{j}=t_{j-1}+p_{j}^{2} / d_{j}, \\
\frac{d_{j}}{}=d_{j} t_{j} / t_{j-1}, \\
\beta_{j}=p_{j} /\left(d_{j} t_{j}\right) .
\end{array}\right\}
$$

Clearly the required Cholesky factors are given by $\bar{L}=L \widetilde{M}$ and $\bar{D}=\widetilde{D}$. These recurrence relations for computing $\beta_{j}$ and $\bar{d}_{j}$ are identical to those given by Fletcher and Powell (1973) although they have been derived in a different way.

The special structure of the matrix $\widetilde{M}$ enables the product $L \widetilde{M}$ to be efficiently computed in terms of the $\beta_{j}$ using the following forward recurrence relations suggested by Gill, Golub, Murray and Saunders (1974):

(i) define $v^{(1)}=L p$;

(ii) for $j=1,2, \ldots, m$ set

$$
\left.\begin{array}{rl}
v_{r}^{(j+1)} & =v_{r}^{(j)}-p_{j} l_{r j} \\
\bar{l}_{r j} & =l_{r j}+\beta_{j} v_{r}^{(j+1)}
\end{array}\right\}, \quad r=j+1, \ldots, m .
$$

The vector $v^{(1)}$ needed to initialize the recurrence relations is known, since $v^{(1)}=$ $L p=v$. Also, each of the vectors $v^{(j)}(j=1,2, \ldots, m)$ can be obtained during the $j$ th stage of the initial forward substitution (20) since

$$
v_{r}^{(j)}=\sum_{i=j}^{m} l_{r i} p_{i}=v_{r}-\sum_{i=1}^{j-1} l_{r i} p_{i}, \quad r=j, j+1, \ldots, m .
$$

We note also that, using the expression for $v_{r}^{(j+1)}$, we can rearrange the equation for $\bar{l}_{r j}$ in the form

$$
\begin{aligned}
\bar{l}_{r j} & =l_{r j}+\beta_{j}\left(v_{r}^{(j)}-p_{j} l_{r j}\right)=\left(1-p_{j} \beta_{j}\right) l_{r j}+\beta_{j} v_{r}^{(j)} \\
& =\left(d_{j} / \bar{d}_{j}\right) l_{r j}+\beta_{j} v_{r}^{(j)} .
\end{aligned}
$$

This method requires $3 m^{2} / 2+O(m)$ multiplications to completely update the factors, whereas only $m^{2}+O(m)$ are required using (19) with (20).

Rounding-error analysis of the recurrence relations (19) and (20) and of (19) and (21) have been carried out by Fletcher and Powell (1973). This analysis shows that the corresponding rounding errors involve a term with coefficient $\bar{d}_{j} / d_{j}$ and $d_{j} / \bar{d}_{j}$, respectively. The recurrence relations (19ii) indicate that $\bar{d}_{j}>d_{j}$ for all $j$; and, consequently, the formula (21) should be used to obtain the new factor, since the term $d_{j} / \bar{d}_{j}$ has a damping effect on the error. The resulting algorithm has the unsatisfactory feature that an additional $\mathrm{m}^{2} / 2$ multiplications are required. However, Gentleman (1973) has suggested using formula (20) until the ratio $\bar{d}_{j} / d_{j}$ exceeds a certain fixed quantity. It has been observed in practice that the amount of work for this modified process is still ap- 
proximately $m^{2}+O(m)$ since large values of $\bar{d}_{j} / d_{j}$ are only likely to occur on one or two occasions during a single updating. For example, if $\bar{d}_{k} / d_{k}$ exceeds the bound, only $m-k$ additional multiplications are required.

In summary, the algorithm for performing the modification (15) is given by

(i) define $t_{0}=1, v^{(1)}=v$;

(ii) for $j=1,2, \ldots, m$ compute

$p_{j}=v_{j}^{(j)}$,

$\underline{t_{j}}=t_{j-1}+p_{j}^{2} / d_{j}$,

$\overline{d_{j}}=d_{j} t_{j} / t_{j-1}$,

$\beta_{j}=p_{j} /\left(d_{j} t_{j}\right)$,

if $\bar{d}_{j} / d_{j}>4$, then set

$$
\left.\begin{array}{rl}
\bar{l}_{r j} & =\left(t_{j-1} / t_{j}\right) l_{r j}+\beta_{j} v_{r}^{(j)} \\
v_{r}^{(j+1)} & =v_{r}^{(j)}-p_{j} l_{r j}
\end{array}\right\}, \quad r=j+1, \ldots, m,
$$

otherwise set

$$
\left.\begin{array}{rl}
v_{r}^{(j+1)} & =v_{r}^{(j)}-p_{j} l_{r j} \\
\bar{l}_{r j} & =l_{r j}+\beta_{j} v_{r}^{(j+1)}
\end{array}\right\}, \quad r=j+1, \ldots, m .
$$

5.2. $\bar{L} \bar{D} \bar{L}^{T}=L D L^{T}-v v^{T}$. In this case, instead of (17) we have

$$
\bar{A}=L\left(D-p p^{T}\right) L^{T}
$$

where $p$ satisfies (18). Consider the quantity $\alpha^{2}=1-p^{T} D^{-1} p$. From (22) we have

$$
\operatorname{det}(\bar{A})=[\operatorname{det}(L)]^{2} \operatorname{det}\left(D-p p^{T}\right)
$$

Since $L$ is unit lower triangular $\operatorname{det}(L)=1$, and consequently

$$
\operatorname{det}(\bar{A})=\operatorname{det}\left(D-p p^{T}\right)=\alpha^{2} \operatorname{det}(D) \text {. }
$$

Since by assumption $\bar{A}$ is positive definite, $\operatorname{det}(\bar{A})>0$ and $\alpha^{2}$ is positive. This implies that we can apply Lemma A4 to give the factorization $D-p p^{T}=\widetilde{M} \widetilde{D} \widetilde{M}^{T}$, using the recurrence relations:

(i) define $t_{m+1}=\alpha^{2}$;

(ii) for $j=m, m-1, \ldots, 1$ set

$$
t_{j}=t_{j+1}+p_{j}^{2} / d_{j}, \quad \overline{d_{j}}=d_{j} t_{j+1} / t_{j}, \quad \beta_{j}=-p_{j} /\left(d_{j} t_{j+1}\right) .
$$

Since the elements of the vector $\beta$ are computed in the order $\beta_{m}, \beta_{m-1}, \ldots, \beta_{1}$, it is convenient to compute the product $L \widetilde{M}$ using the backward recurrence relations:

$$
\text { for } j=m, m-1, \ldots, 1 \text { set }
$$

$$
\left.\begin{array}{rl}
v_{j}^{(j)} & =p_{j}, \\
\bar{l}_{r j} & =l_{r j}+\beta_{j} v_{r}^{(j+1)} \\
v_{r}^{(j)} & =v_{r}^{(j+1)}+p_{j} l_{r j}
\end{array}\right\} ; \quad r=j+1, \ldots, m .
$$

In this case there is no need to consider an alternative recurrence relation for $\bar{l}_{r j}$ 
since, as mentioned earlier in 5.1, the error involved using a recurrence relation of the form (23) is multiplied by the factor $\bar{d}_{j} / d_{j}$ and $\bar{d}_{j} \leqslant d_{j}$ for all $j$.

Unlike the recurrence relations for adding a rank-one matrix, the formation of $\bar{L}$ cannot take place during the computation of the vectors $p$ and $\beta$ since all of $p$ must be known before the recurrence relations for $\beta$ can commence. For this reason the computation of the modified factors requires $3 \mathrm{~m}^{2} / 2+O(m)$ multiplications. It is a feature of this method that, provided $\alpha^{2}>0$, the modified matrix is positive definite regardless of any rounding errors made.

The final algorithm to perform the modification (16) is thus as follows:

(i) Solve the equations $L p=v$ and define $t_{m+1}=1-p^{T} D^{-1} p$; if $t_{m+1} \leqslant 0$ set $t_{m+1}=\epsilon$, where $\epsilon(\epsilon>0)$ is the machine precision;

(ii) for $j=m, m-1, \ldots, 1$ set

$$
\begin{aligned}
& t_{j}=t_{j+1}+p_{j}^{2} / d_{j}, \\
& \overline{d_{j}}=d_{j} t_{j+1} / t_{j} \text {, } \\
& \beta_{j}=-p_{j} /\left(d_{j} t_{j+1}\right) \text {, } \\
& v_{j}^{(j)}=p_{j} \text {, } \\
& \left.\begin{array}{c}
\bar{l}_{r j}=l_{r j}+\beta_{j} v_{r}^{(j+1)} \\
v_{r}^{(j)}=v_{r}^{(j+1)}+p_{j} l_{r j}
\end{array}\right\}, \quad r=j+1, \ldots, m .
\end{aligned}
$$

Acknowledgments. The authors would like to thank Dr. J. H. Wilkinson for his careful reading of the manuscript and a number of helpful suggestions.

Appendix. Here we give the lemmas and theorems referred to earlier which develop the special structure of the following matrices:

(a) the product $P$ of certain sequences of elementary orthogonal matrices which reduce an $n$-vector $z$ to a multiple of the unit vector $e_{n}$, thus:

$$
P z=\|z\| e_{n}
$$

with $P=P_{n-1} P_{n-2} \cdots P_{2} P_{1}$ and $P=P_{1} P_{2} \cdots P_{n-2} P_{n-1}$, where each $P_{j}$ is a plane rotation;

(b) the $L Q$ factors of matrices of the form

$$
\left[\begin{array}{ll}
I & p \\
& 1
\end{array}\right] \text { and } I-q q^{T} \quad(\|q\|=1)
$$

(c) the $L D V$ factors of matrices of the form

$$
\left[\begin{array}{ll}
D & p \\
& 1
\end{array}\right] \text { and } D-q q^{T} \quad\left(\left\|D^{-1 / 2} q\right\|=1\right)
$$

where $D$ is a positive-definite diagonal matrix;

(d) the Cholesky factors of matrices of the form

$$
D+p p^{T} \text { and } D-p p^{T} .
$$

Lemma A1. Let $z$ be an n-vector and $P$ an orthogonal matrix such that

$$
P z=\|z\| e_{n} .
$$


In particular, let $P$ be the product of plane rotations

$$
P=P_{n-1} \cdots P_{2} P_{1},
$$

where each $P_{j}$ is a symmetric orthogonal matrix of the form

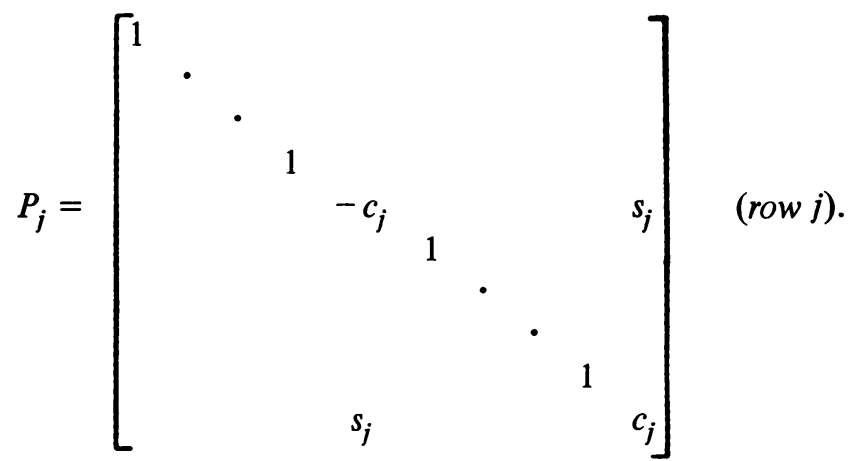

Equation (1) holds if the elements $c_{j}$ and $s_{j}$ are such that

$$
\left[\begin{array}{cc}
-c_{j} & s_{j} \\
s_{j} & c_{j}
\end{array}\right]\left[\begin{array}{c}
z_{j} \\
\rho_{j-1}
\end{array}\right]=\left[\begin{array}{c}
0 \\
\rho_{j}
\end{array}\right],
$$

where

$$
\begin{aligned}
\rho_{j}^{2} & =\rho_{j-1}^{2}+z_{j}^{2}, \\
c_{j} & =\rho_{j-1} / \rho_{j}, \\
s_{j} & =z_{j} / \rho_{j},
\end{aligned}
$$

for $j=1,2, \ldots, n-1$. (When $j=1$ we define $\rho_{0}=z_{n}$.) If the last component of $z$ is nonzero, $P$ can be formed into the matrix

$$
P=\left[\begin{array}{cccccc}
\gamma_{1} & & & & & \sigma_{1} z_{n} \\
\sigma_{2} z_{1} & \gamma_{2} & & & & \sigma_{2} z_{n} \\
\sigma_{3} z_{1} & \sigma_{3} z_{2} & \gamma_{3} & & & \sigma_{3} z_{n} \\
\cdot & \cdot & \cdot & \cdot & & \cdot \\
\cdot & \cdot & \cdot \cdot \cdot & & \cdot \\
\cdot & \cdot & \cdot \cdot \cdot \cdot & & \cdot \\
\sigma_{n-1} z_{1} & \sigma_{n-1} z_{2} & \cdot \cdot \cdot \cdot & \gamma_{n-1} & \sigma_{n-1} z_{n} \\
\sigma_{n} z_{1} & \sigma_{n} z_{2} & \cdot \cdot \cdot \cdot \cdot \sigma_{n-1} z_{n-1} & \sigma_{n} z_{n}
\end{array}\right],
$$

where the elements $\sigma_{j}$ and $\gamma_{j}$ are defined by the recurrence relations

$$
\left.\begin{array}{c}
\text { 4(i) for } j=1,2, \ldots, n-1 \text { define } \\
\sigma_{j}=s_{j} / \rho_{j-1}, \quad \gamma_{j}=-c_{j} ; \\
\text { 4(ii) define } \sigma_{n}=1 / \rho_{n-1} \quad(=1 /\|z\|) .
\end{array}\right\}
$$

[Note: We require $z_{n} \neq 0$, but in general there may be $z_{j}=0$ for $j<n$. For such $j$ we define $P_{j}=I, \sigma_{j}=0, \gamma_{j}=1$, so the $j$ th row and column of $P$ will be unit vectors. Without loss of generality we may assume $z_{1} \neq 0$.] 
Proof. We shall define

and the partial product

$$
P=P_{n-1} P_{n-2} \cdots P_{2} P_{1} \equiv\left[\begin{array}{c}
p_{1}^{T} \\
p_{2}^{T} \\
\cdot \\
\cdot \\
\cdot \\
p_{n}^{T}
\end{array}\right],
$$

$$
P_{t} P_{t-1} \cdots P_{2} P_{1} \equiv Q_{t}
$$

The first $t$ rows of $Q_{t}$ are unaffected by subsequent rotations $P_{t+1}, \ldots, P_{n-1}$ and so we can write

(5)

$$
Q_{t}=\left[\begin{array}{c}
p_{1}^{T} \\
\cdot \\
\cdot \\
p_{t}^{T} \\
e_{t+1}^{T} \\
\cdot \\
\cdot \\
\cdot \\
e_{n-1}^{T} \\
q_{t}^{T}
\end{array}\right]
$$

Using Eq. (1), the vector $Q_{t} z \equiv v_{t}$ is of the form

$$
v_{t}^{T}=\left(0, \ldots, 0, z_{t+1}, \ldots, z_{n-1}, \rho_{t}\right),
$$

and since $Q_{t}$ is orthogonal

$$
z=Q_{t}^{T} v_{t}
$$

Substituting (5) and (6) into (7), we have

$$
\left[\begin{array}{c}
z_{1} \\
\cdot \\
\cdot \\
z_{t} \\
z_{t+1} \\
\cdot \\
\cdot \\
z_{n-1} \\
z_{n}
\end{array}\right]=\left[\begin{array}{c}
0 \\
\cdot \\
\cdot \\
z_{t+1} \\
\cdot \\
\cdot \\
z_{n-1} \\
0
\end{array}\right]+\rho_{t} q_{t}
$$


giving

$$
q_{t}^{T}=\left(\frac{z_{1}}{\rho_{t}}, \frac{z_{2}}{\rho_{t}}, \ldots, \frac{z_{t}}{\rho_{t}}, 0, \ldots, 0, \frac{z_{n}}{\rho_{t}}\right) .
$$

At the $(t+1)$ th stage, $Q_{t}$ is premultiplied by $P_{t+1}$ giving the row $p_{t+1}^{T}$ as a linear combination of the two rows

$$
\begin{gathered}
(0,0, \ldots, 0,1,0, \ldots, 0,0) \\
\left(\frac{z_{1}}{\rho_{t}}, \frac{z_{2}}{\rho_{t}}, \ldots, \frac{z_{t}}{\rho_{t}}, 0,0, \ldots, 0, \frac{z_{n}}{\rho_{t}}\right) .
\end{gathered}
$$

Thus

$$
p_{t+1}^{T}=\left(\frac{z_{1} s_{t+1}}{\rho_{t}}, \frac{z_{2} s_{t+1}}{\rho_{t}}, \ldots, \frac{z_{t} s_{t+1}}{\rho_{t}},-c_{t+1}, 0, \ldots, \frac{z_{n} s_{t+1}}{\rho_{t}}\right)
$$

and if we define $\sigma_{t+1}=s_{t+1} / \rho_{t}$ and $\gamma_{t+1}=-c_{t+1}$, we have the required result.

Theorem A1 ( $L Q$ FACTORIzATION OF AN ElemEnTARY MATRIX). Let $\widetilde{A}$ be a matrix of the form

$$
\widetilde{A}=\left[\begin{array}{cc}
I & q \\
& 1
\end{array}\right] \equiv I_{m+1}+\left[\begin{array}{l}
q \\
0
\end{array}\right] e_{m+1}^{T}
$$

where $q$ is an m-vector. The matrix $\widetilde{A}$ has the $L Q$ factorization $\widetilde{A}=\widetilde{L} \widetilde{Q}$, where $\widetilde{L}$ is a special lower-triangular matrix and $\widetilde{Q}$ is an orthogonal matrix of the form

$$
\widetilde{L}=\left[\begin{array}{cc}
\widetilde{M} & \\
\sigma^{T} & \alpha
\end{array}\right], \quad \widetilde{Q}=\left[\begin{array}{cc}
\widetilde{N} & \sigma \\
-\alpha q^{T} & \alpha
\end{array}\right] .
$$

Both $\widetilde{M}$ and $\widetilde{N}$ are special lower-triangular matrices defined by

$$
\widetilde{M}=\widetilde{M}(q, \sigma, \delta), \quad \widetilde{N}=\widetilde{N}(\sigma,-q, \gamma)=\widetilde{M}^{-1}=\widetilde{M}^{T}-\sigma q^{T},
$$

where the vectors $\sigma, \gamma, \delta$ and the scalar $\alpha$ are generated by the following recurrence relations:

$$
\left.\begin{array}{rl}
\text { 8(i) } & \text { define } \rho_{0}=1 ; \\
\text { 8(ii) } & \text { for } j=1,2, \ldots, \text { m set } \\
& \rho_{j}^{2}=\rho_{j-1}^{2}+q_{j}^{2}, \\
& \sigma_{j}=-q_{j} /\left(\rho_{j} \rho_{j-1}\right), \\
& \gamma_{j}=-\rho_{j-1} / \rho_{j}, \\
& \delta_{j}=1 / \gamma_{j} ; \\
\text { 8(iii) define } \alpha=1 / \rho_{m} .
\end{array}\right\}
$$

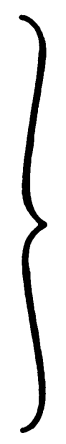

Proof (of Theorem A1). The $L Q$ factors of $\widetilde{A}$ could be computed directly from the relation

$$
\widetilde{A} \widetilde{Q}^{T} \equiv\left[\begin{array}{rr}
I & q \\
& 1
\end{array}\right] \widetilde{Q}^{T}=\widetilde{L}
$$


where $\widetilde{Q}$ is a product of plane rotations designed to eliminate the elements of $q$ one by one. However, we show now that $\widetilde{Q}$ may instead be constructed as a product of plane rotations such that

$$
\widetilde{Q}\left[\begin{array}{c}
-q \\
1
\end{array}\right] \equiv P_{m} \cdots P_{2} P_{1}\left[\begin{array}{c}
-q \\
1
\end{array}\right]=\omega e_{m+1}
$$

with

$$
\omega=\left(q^{T} q+1\right)^{1 / 2}
$$

(It turns out that this method is slightly more efficient, and it allows us to use Lemma A1 to develop the structure of $\widetilde{Q}$.) Let $\widetilde{L}$ be partitioned in the form

$$
\widetilde{L}=\left[\begin{array}{ll}
\widetilde{M} & \\
y^{T} & \alpha
\end{array}\right],
$$

and suppose that in place of (9) and (10) we have

$$
\widetilde{Q}\left[\begin{array}{c}
-q \\
1
\end{array}\right]=\left[\begin{array}{l}
w \\
\omega
\end{array}\right], \quad w^{T} w+\omega^{2}=q^{T} q+1
$$

Multiplying the relation $\widetilde{L} \widetilde{Q}=\widetilde{A}$ by $\left[\begin{array}{c}-q \\ 1\end{array}\right]$ gives

$$
\left[\begin{array}{ll}
\widetilde{M} & \\
y^{T} & \alpha
\end{array}\right]\left[\begin{array}{l}
w \\
\omega
\end{array}\right]=\left[\begin{array}{ll}
I & q \\
& 1
\end{array}\right]\left[\begin{array}{c}
-q \\
1
\end{array}\right]
$$

i.e.

$$
\left[\begin{array}{c}
\tilde{M} w \\
y^{T} w+\alpha \omega
\end{array}\right]=\left[\begin{array}{l}
0 \\
1
\end{array}\right]
$$

Since $\widetilde{M}$ must be nonsingular this gives $w=0, \alpha \omega=1$ and $\omega^{2}=q^{T} q+1$.

We have thus proved that Eqs. (9) and (10) are true.

From Lemma A1 we can therefore say that $\widetilde{Q}=P_{m} \cdots P_{2} P_{1}$ is of the form

$$
\widetilde{Q}=\left[\begin{array}{cc}
\widetilde{N} & \sigma \\
-\alpha q^{T} & \alpha
\end{array}\right]
$$

where

(i) $\tilde{N}=\widetilde{N}(\sigma,-q, \gamma)$ is a special lower-triangular matrix;

(ii) the quantities $\sigma, \gamma$ and $\alpha$ are obtained from the recurrence relations (3) and (4) by replacing $z, \sigma$ and $n$ by $\left[\begin{array}{c}-q \\ 1\end{array}\right],\left[\begin{array}{c}\sigma \\ \alpha\end{array}\right]$ and $m+1$, respectively;

(iii) in particular,

$$
\alpha \equiv \sigma_{m+1}=1 /\left\|\left[\begin{array}{c}
-q \\
1
\end{array}\right]\right\|=\left(q^{T} q+1\right)^{-1 / 2}=1 / \omega,
$$

which is consistent with the use of $\alpha$ in $\widetilde{L}$ above.

Using (3) to eliminate $c_{j}$ and $s_{j}$ in (4) now gives the recurrence relations (8) for generating $\sigma, \gamma$ and $\alpha$, and the structure of $\widetilde{Q}$ and $\widetilde{N}$ is determined.

It remains to determine the structure of $\widetilde{L}$ and $\widetilde{M}$. From the equation $\widetilde{A}=\widetilde{L} \widetilde{Q}$ it follows immediately that $\widetilde{M} \widetilde{N}=I$, and hence the diagonals of $\widetilde{M}$ are the reciprocals 
of those of $\widetilde{N}$, i.e. $\widetilde{M}_{i i}=1 / \gamma_{j}=\delta_{j}$. Next, the equation $\widetilde{A} \widetilde{Q}^{T}=\widetilde{L}$ readily gives the relation $\widetilde{M}=\widetilde{N}^{T}+q \sigma^{T}$, and from the structure of $\widetilde{N}$ it follows that $\widetilde{M}_{i j}=q_{i} \sigma_{j}$ for $i>j$, and hence $\widetilde{M}$ is the special lower-triangular matrix $\widetilde{M}(q, \sigma, \delta)$. Finally, the equation $\widetilde{A} \widetilde{Q}^{T}=\widetilde{L}$ also gives $y=\sigma$, which completes the structure of $\widetilde{L}$. The theorem is now proved.

Theorem A2 (LDV FACtorization of AN ElEmEntARy MATrix). Let $A$ be $a$ matrix of the form

$$
A=D+p e_{s}^{T},
$$

where $p$ is an m-vector such that $p_{s} \neq 0$ and $D=\operatorname{diag}\left(d_{1}, d_{2}, \ldots, d_{m}\right)$ with $d_{s}=0$ and $d_{j} \geqslant 0$ for $j=1,2, \ldots, s-1, s+1, \ldots, m$. If $p_{s}$ is the first element of $p$ such that $\left|p_{s}\right|>0$ and $d_{s}=0$, then $A$ has an $L D V$ factorization $A=\widetilde{L} \widetilde{D} \widetilde{V}$ where

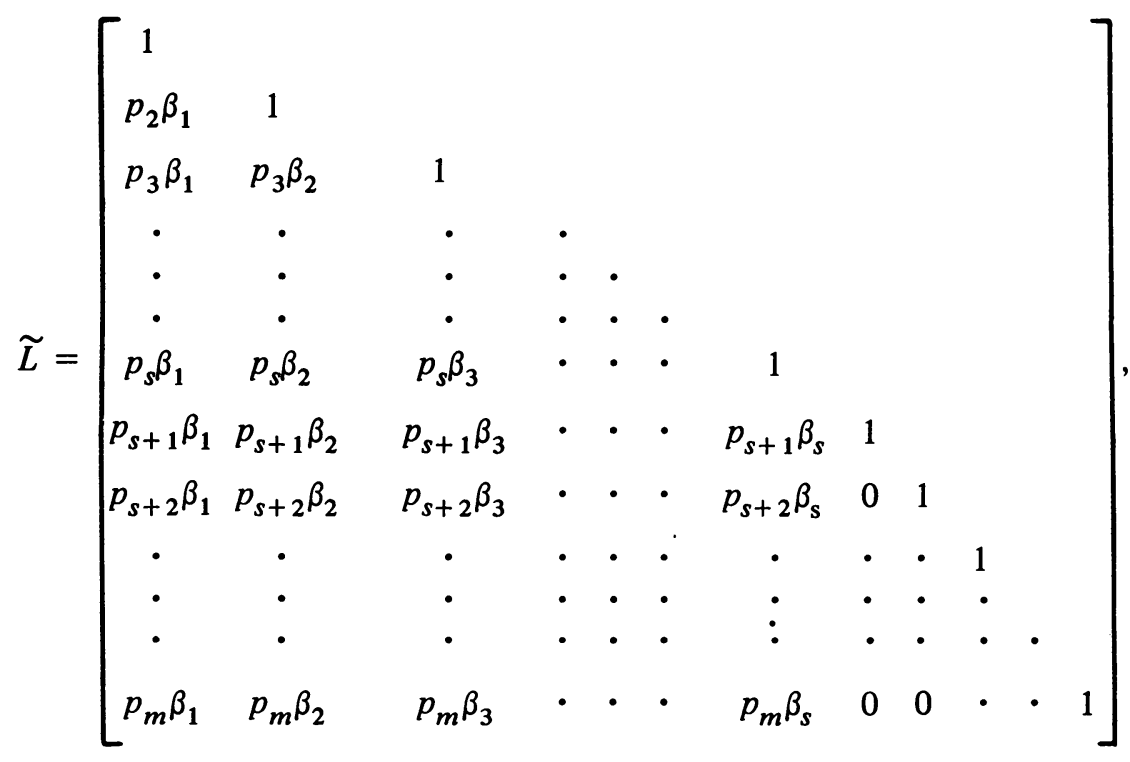

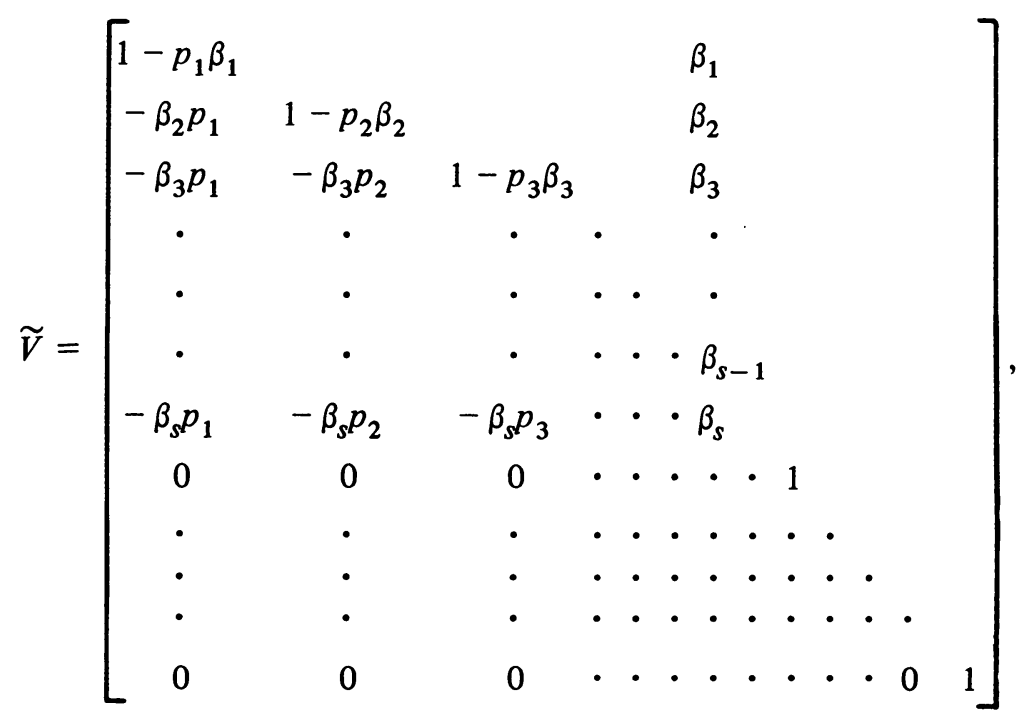




$$
\widetilde{D}=\operatorname{diag}\left(\widetilde{d_{1}}, \widetilde{d}_{2}, \ldots, \widetilde{d}_{m}\right)=\operatorname{diag}\left(\hat{d}_{1}, \hat{d}_{2}, \ldots, \hat{d}_{s-1}, \hat{d}_{s}, d_{s+1}, \ldots, d_{m}\right)
$$

and the matrix $\left(\widetilde{D}^{+}\right)^{1 / 2} \widetilde{V}\left(D^{+}\right)^{-1 / 2}$ is orthogonal. The vectors $\hat{d}$ and $\beta$ are generated by the following recurrence relations:

$$
\left.\begin{array}{rl}
\text { 11(i) define } t_{0}=1 \\
11(\text { ii) } & \text { for } k=1,2, \ldots, s-1 \text { compute the following: } \\
& \text { if } d_{k}=0 \text { then set } \nu_{k}=0 \text { otherwise set } \nu_{k}=p_{k} / d_{k}, \\
& t_{k}=t_{k-1}+\nu_{k} p_{k}, \\
& \hat{d}_{k}=d_{k} t_{k} / t_{k-1}, \\
& \beta_{k}=v_{k} / t_{k} ; \\
11 \text { (iii) define } \hat{d}_{s}=p_{s}^{2} / t_{s-1} \text { and } \beta_{s}=1 / p_{s} .
\end{array}\right\}
$$

Proof. We shall prove this theorem in two stages. Firstly, we shall assume that $d_{s}$ is the only zero element of $D$ and then consider the case where other $d_{j}$ are zero (together with their associated $p_{j}$ ).

Consider the matrix $A_{s}$ made up of the first $s$ rows and columns of $A$. If $A$ is partitioned as

$$
A=\left[\begin{array}{ccc}
D_{1} & p^{(1)} & \\
& p_{s} & \\
& p^{(2)} & D_{2}
\end{array}\right],
$$

then $A_{s}$ can be written as

$$
\begin{aligned}
A_{s}=\left[\begin{array}{ll}
D_{1} & p^{(1)} \\
& p_{s}
\end{array}\right] & =\left[\begin{array}{ll}
I & \\
& p_{s}
\end{array}\right]\left[\begin{array}{ll}
D_{1}^{1 / 2} & \\
& 1
\end{array}\right]\left[\begin{array}{ll}
I & q \\
& 1
\end{array}\right]\left[\begin{array}{ll}
D_{1}^{1 / 2} & \\
& 1
\end{array}\right] \\
& =\left[\begin{array}{ll}
I & \\
& p_{s}
\end{array}\right]\left(D_{s}^{+}\right)^{1 / 2} \widetilde{A}_{s}\left(D_{s}^{+}\right)^{1 / 2},
\end{aligned}
$$

where

$$
\widetilde{A}_{s}=\left[\begin{array}{cc}
I & q \\
& \\
& 1
\end{array}\right], \quad D_{s}^{+}=\left[\begin{array}{cc}
D_{1} & \\
& \\
& 1
\end{array}\right] \text { and } q=D_{1}^{-1 / 2} p .
$$

From Theorem A1 we know that $\widetilde{A}_{s}$ has the orthogonal factorization $\widetilde{A}_{s}=\widetilde{L}_{s} \widetilde{Q}_{s}$ where $\widetilde{L}_{s}$ and $\widetilde{Q}_{s}$ are constructed from the quantities $q, \sigma, \gamma, \delta$ and $\alpha$ as shown. Let us define

$$
\left.\begin{array}{l}
\overline{d_{j}}=d_{j} \delta_{j}^{2}, \quad \beta_{j}=\sigma_{j} /\left(\delta_{j} d_{j}^{1 / 2}\right), \\
\theta_{j}=\gamma_{j} / \delta_{j}=1 / \delta_{j}^{2}, \quad \Delta=\operatorname{diag}\left(\delta_{1}, \delta_{2}, \ldots, \delta_{s-1}, \alpha\right),
\end{array}\right\}
$$

and $e=(1,1, \ldots, 1)^{T}$. Using the notation of Theorem A1, we now have 


$$
A_{s}=\left[\begin{array}{ll}
I & \\
& p_{s}
\end{array}\right]\left(D_{s}^{+}\right)^{1 / 2} \widetilde{A}_{s}\left(D_{s}^{+}\right)^{1 / 2}=\left[\begin{array}{ll}
I & \\
& \\
& p_{s}
\end{array}\right]\left(D_{s}^{+}\right)^{1 / 2} \widetilde{L}_{s} \widetilde{Q}_{s}\left(D_{s}^{+}\right)^{1 / 2},
$$

where

$$
\begin{aligned}
{\left[\begin{array}{ll}
I & \\
& p_{s}
\end{array}\right]\left(D_{s}^{+}\right)^{1 / 2} \widetilde{L}_{s} } & =\left[\begin{array}{ll}
I & \\
& p_{s}
\end{array}\right]\left(D_{s}^{+}\right)^{1 / 2}\left[\begin{array}{cc}
\widetilde{M}(q, \sigma, \delta) & \\
\sigma^{T} & \alpha
\end{array}\right] \\
& =\left[\begin{array}{ll}
\widetilde{M}(p, \beta, e) \\
p_{s} \beta^{T} & 1
\end{array}\right]\left(D_{s}^{+}\right)^{1 / 2} \Delta\left[\begin{array}{ll}
I \\
\\
p_{s}
\end{array}\right]=\hat{L}\left(D_{s}^{+}\right)^{1 / 2} \Delta\left[\begin{array}{ll}
I & \\
& p_{s}
\end{array}\right],
\end{aligned}
$$

and

$$
\widetilde{Q}_{s}\left(D_{s}^{+}\right)^{1 / 2}=\left[\begin{array}{cc}
\widetilde{N}(\sigma,-q, \gamma) & \sigma \\
-\alpha q^{T} & \alpha
\end{array}\right]\left(D_{s}^{+}\right)^{1 / 2}
$$

(14b)

$$
=\left[\begin{array}{ll}
I & \\
& p_{s}
\end{array}\right] \Delta\left(D_{s}^{+}\right)^{1 / 2}\left[\begin{array}{cc}
\widetilde{N}\left(\beta,-p^{(1)}, \theta\right) & \beta \\
-\left(\frac{1}{p_{s}}\right) p^{(1) T} & 1 / p_{s}
\end{array}\right]=\left[\begin{array}{ll}
I & \\
& p_{s}
\end{array}\right] \Delta\left(D_{s}^{+}\right)^{1 / 2} \hat{V} .
$$

Combining (14a) and (14b) gives $A_{s}=\hat{L} \hat{D} \hat{V}$, where

$$
\hat{L}=\left[\begin{array}{cc}
\widetilde{M} & \\
p_{s} \beta^{T} & 1
\end{array}\right], \quad \hat{V}=\left[\begin{array}{cc}
\widetilde{N} & \beta \\
-\left(\frac{1}{p_{s}}\right) p^{(1) T} & 1 / p_{s}
\end{array}\right]
$$

and

$$
\begin{aligned}
\hat{D} & =\left(D_{s}^{+}\right)^{1 / 2} \Delta^{2}\left(D_{s}^{+}\right)^{1 / 2}\left[\begin{array}{ll}
I & \\
& p_{s}^{2}
\end{array}\right]=\Delta^{2} D_{s}^{+}\left[\begin{array}{ll}
I & \\
& p_{s}^{2}
\end{array}\right] \\
& =\operatorname{diag}\left(d_{1} \delta_{1}^{2}, d_{2} \delta_{2}^{2}, \ldots, d_{s-1} \delta_{s-1}^{2}, p_{s}^{2} \alpha^{2}\right)
\end{aligned}
$$

Equations (14b) and (15) also give the orthogonal matrix $\widetilde{Q}_{s}$ as

$$
\widetilde{Q}_{s}=\left[\begin{array}{ll}
I & \\
& \\
& p_{s}
\end{array}\right] \Delta\left(D_{s}^{+}\right)^{1 / 2} \hat{V}\left(D_{s}^{+}\right)^{-1 / 2}=\hat{D}^{1 / 2} \hat{V}\left(D_{s}^{+}\right)^{-1 / 2} .
$$

We can now simplify the expressions for $\bar{d}_{j}, \beta_{j}$ and $\theta_{j}$ in (13). From the definitions of $\rho_{j}, \sigma_{j}, \delta_{j}$ and $q_{j}$ in (8) and (12) we have

$$
\begin{gathered}
\rho_{j}^{2}=\rho_{j-1}^{2}+q_{j}^{2}=\rho_{j-1}^{2}+p_{j}^{2} / d_{j} \\
\bar{d}_{j}=d_{j} / \gamma_{j}^{2}=d_{j} \rho_{j}^{2} / \rho_{j-1}^{2}
\end{gathered}
$$




$$
\begin{gathered}
\beta_{j}=\sigma_{j} \gamma_{j} / d_{j}^{1 / 2}=q_{j} /\left(\rho_{j}^{2} d_{j}^{1 / 2}\right)=p_{j} /\left(\rho_{j}^{2} d_{j}\right) ; \\
\theta_{j}=\gamma_{j}^{2}=\rho_{j-1}^{2} / \rho_{j}^{2}=\left(\rho_{j}^{2}-p_{j}^{2} / d_{j}\right) / \rho_{j}^{2}=1-p_{j}^{2} /\left(\rho_{j}^{2} d_{j}\right)=1-p_{j} \beta_{j} .
\end{gathered}
$$

From 8(iii) we also have $\alpha^{2}=1 / \rho_{s-1}^{2}$. Since all these expressions require $\rho_{j}^{2}$ rather than $\rho_{j}$, we can define $t_{j}=\rho_{j}^{2}$ and avoid the computation of all square roots.

If we now consider the factorization of the complete matrix $D+p e_{s}^{T}$, we must have

$$
A=\left[\begin{array}{ll}
\hat{L} & \\
Y & I
\end{array}\right]\left[\begin{array}{ll}
\hat{D} & \\
& D_{2}
\end{array}\right]\left[\begin{array}{ll}
\hat{V} & \\
& I
\end{array}\right],
$$

where $Y$ is a matrix to be determined. If the factors of the last expression are multiplied out and right- and left-hand sides are equated, we have $p^{(2)} e_{s}^{T}=Y \hat{D} \hat{V}$, where $e_{s}^{T}$ is the last row of the sth-order identity matrix. Multiplying both sides by $\left(D_{s}^{+}\right)^{-1} \hat{V}^{T}$ and noting that $\hat{V}\left(D_{s}^{+}\right)^{-1} \hat{V}^{T}=\hat{D}^{-1}$, since $\hat{D}^{1 / 2} \hat{V}\left(D_{s}^{+}\right)^{-1 / 2}$ is orthogonal, we have

$$
p^{(2)} e_{s}^{T}\left(D_{s}^{+}\right)^{-1} \hat{V}^{T}=Y \hat{D} \hat{V}\left(D_{s}^{+}\right)^{-1} \hat{V}^{T}=Y .
$$

Consequently, since

$$
e_{s}^{T}\left(D_{s}^{+}\right)^{-1}=e_{s}^{T} \text { and } e_{s}^{T} \hat{V}^{T}=\left[\begin{array}{ll}
\beta^{T} & \frac{1}{p_{s}}
\end{array}\right]
$$

we have

$$
Y=p^{(2)}\left[\begin{array}{ll}
\beta^{T} & \frac{1}{p_{s}}
\end{array}\right] .
$$

If we define $\beta_{s}=1 / p_{s}$, this completes the proof in the case where $d_{j}>0$ for $j=1$, $2, \ldots, s-1, s+1, \ldots, m$.

If $A$ has $k$ rows and columns equal to zero (that is $d_{j}=0$ corresponding to $p_{j}=0$ ), we can apply the method just described to the matrix of $m-k$ remaining rows and columns and regard the $L D V$ factors so obtained as being of order $m$ by inserting suitable rows and columns of the identity matrix. This gives the recurrence relations (11).

Corollary. Let $A$ be a matrix of the form

$$
A=D+\left[\begin{array}{l}
p \\
0
\end{array}\right] e_{m+1}^{T} \equiv\left[\begin{array}{ll}
D_{1} & p \\
& 1
\end{array}\right]
$$

where $p$ is an m-vector and

$$
D=\operatorname{diag}\left(d_{1}, d_{2}, \ldots, d_{m}, 1\right) \equiv\left[\begin{array}{ll}
D_{1} & \\
& \\
& 1
\end{array}\right],
$$

with $D_{1}$ positive definite. The matrix $A$ has an $L D V$ factorization $A=\hat{L} \hat{D} \hat{V}$ where

$$
\hat{L}=\left[\begin{array}{ll}
\widetilde{M} & \\
\beta^{T} & 1
\end{array}\right], \quad \hat{D}=\operatorname{diag}\left(\bar{d}_{1}, \bar{d}_{2}, \ldots, \bar{d}_{m}, \alpha^{2}\right), \quad \hat{V}=\left[\begin{array}{cc}
\widetilde{N} & \beta \\
-p^{T} & 1
\end{array}\right],
$$


and the matrix $\hat{D}^{1 / 2} \hat{V} D^{-1 / 2}$ is orthogonal. Both $\widetilde{M}$ and $\widetilde{N}$ are special lower-triangular matrices defined by

$$
\widetilde{M}=\widetilde{M}(p, \beta), \quad \widetilde{N}=\widetilde{N}(\beta,-p, \theta)=\widetilde{M}^{T}-\beta p^{T},
$$

where the vectors $\bar{d}, \beta, \theta$ and the scalar $\alpha^{2}$ are generated by the following recurrence relations:

$$
\left.\begin{array}{cl}
\text { 16(i) } & \text { define } t_{0}=1 \\
16(\mathrm{ii}) & \text { for } j=1,2, \ldots, m \text { set } \\
& t_{j}=t_{j-1}+p_{j}^{2} / d_{j}, \\
& \bar{d}_{j}=d_{j} t_{j} / t_{j-1}, \\
& \beta_{j}=p_{j} /\left(d_{j} t_{j}\right), \\
& \theta_{j}=1-p_{j} \beta_{j} ; \\
16\left(\text { iii) define } \alpha^{2}=1 / t_{m} . \square\right.
\end{array}\right\}
$$

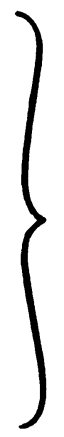

Lemma A2. Let $z$ be an n-vector and $P$ an orthogonal matrix such that

$$
P z=\|z\| e_{n} .
$$

In particular, let $P$ be the product of plane rotations $P=P_{1} P_{2} \cdots P_{n-1}$, where each $P_{j}$ is the form given in Lemma A1. Equation (17) holds if the element $c_{j}$ and $s_{j}$ defining $P_{j}$ are such that

$$
\left[\begin{array}{cc}
-c_{j} & s_{j} \\
s_{j} & c_{j}
\end{array}\right]\left[\begin{array}{c}
z_{j} \\
\rho_{j+1}
\end{array}\right]=\left[\begin{array}{l}
0 \\
\rho_{j}
\end{array}\right],
$$

where

$$
\rho_{j}^{2}=\rho_{j+1}^{2}+z_{j}^{2}, \quad c_{j}=\rho_{j+1} / \rho_{j}, \quad s_{j}=z_{j} / \rho_{j},
$$

for $j=n-1, n-2, \ldots, 1$. (When $j=n-1$ we define $\rho_{n}=z_{n}$.) If the last component of $z$ is nonzero, $P$ can be formed into the matrix

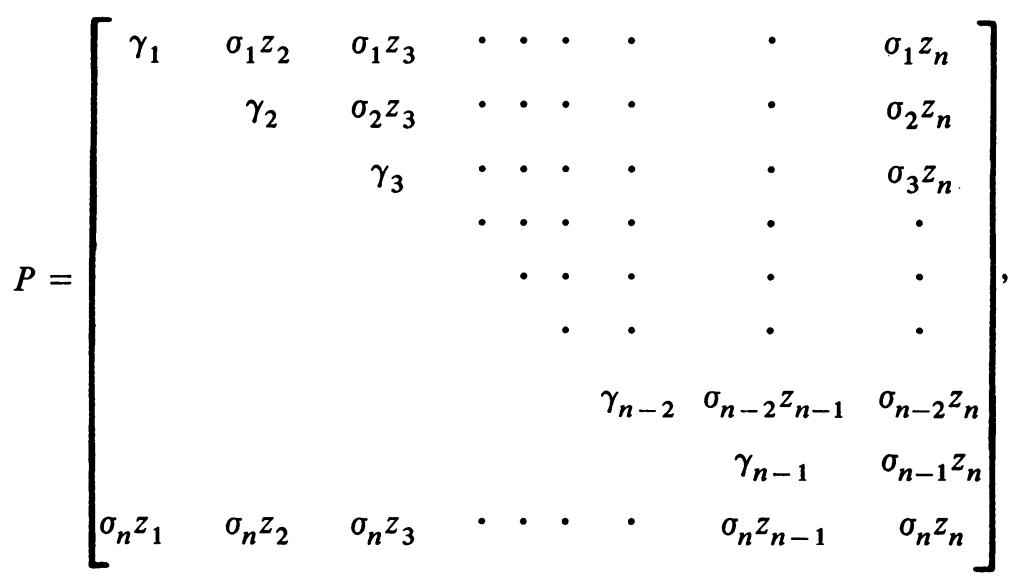

where the elements $\sigma_{j}$ and $\gamma_{j}$ are defined by the recurrence relations 


$$
\begin{aligned}
& \text { 19(i) for } j=n-1, n-2, \ldots, 1 \text { define } \\
& \sigma_{j}=s_{j} / \rho_{j+1}, \quad \gamma_{j}=-c_{j} \text {; } \\
& \text { 19(ii) define } \sigma_{n}=1 / \rho_{1} \quad(=1 /\|z\|) \text {. }
\end{aligned}
$$

[Note: As in Lemma A1, we require $z_{n} \neq 0$; but if $z_{j}=0$ for $j<n$, we define $P_{j}=I$, $\left.\sigma_{j}=0, \gamma_{j}=1.\right]$

Proof. This lemma is proved in a similar way to Lemma A1.

THEOREM A3 ( $L Q$ FACTORIZATION OF AN ELEMENTARY MATRIX). Let $\widetilde{A}$ be an $(m+1) \times(m+1)$ matrix of the form $\widetilde{A}=I_{m+1}-\hat{q} \hat{q}^{T}$, where $\hat{q}=\left[\begin{array}{l}q \\ \alpha\end{array}\right]$, with $\alpha a$ scalar $(\alpha \neq 0)$ and $\|\hat{q}\|=1$. The matrix $\widetilde{A}$ has the $L Q$ factorization $\widetilde{A}=\widetilde{L} \widetilde{Q}$, where $\widetilde{L}$ is a special lower-triangular matrix and $\widetilde{Q}$ is an orthogonal matrix of the form

$$
\widetilde{L}=\left[\begin{array}{cc}
\widetilde{M} & \\
\alpha \sigma^{T} & 0
\end{array}\right], \quad \widetilde{Q}=\left[\begin{array}{cc}
\widetilde{M}^{T} & \alpha \sigma \\
q^{T} & \alpha
\end{array}\right] .
$$

The matrix $\widetilde{M}=\widetilde{M}(q, \sigma, \gamma)$ is a special lower-triangular matrix with the vectors $\sigma$ and $\gamma$ defined by the following recurrence relations:

$$
\left.\begin{array}{rl}
20 \text { (i) } & \text { define } \rho_{m+1}=\alpha ; \\
20(\text { ii) }) & \text { for } j=m, m-1, \ldots, 1 \text { set } \\
& \rho_{j}^{2}=\rho_{j+1}^{2}+q_{j}^{2}, \\
& \sigma_{j}=q_{j} /\left(\rho_{j+1} \rho_{j}\right), \\
& \gamma_{j}=-\rho_{j+1} / \rho_{j} .
\end{array}\right\}
$$

Proof. We shall obtain the $L Q$ factorization of $\widetilde{A}$ by construction. Let $\widetilde{Q}$ be the orthogonal matrix $\widetilde{Q}=P_{1} P_{2} \cdots P_{m}$ constructed as in Lemma A2 such that

$$
\widetilde{Q} \hat{q}=\|\hat{q}\| e_{m+1}=e_{m+1} .
$$

Replacing $z, \sigma$ and $n$ by $\left[\begin{array}{c}q \\ \alpha\end{array}\right],\left[\begin{array}{c}\sigma \\ \sigma_{m+1}\end{array}\right]$ and $m+1$, respectively, we see from Lemma A2 that $\widetilde{Q}$ may be partitioned in the form

$$
\widetilde{Q}=\left[\begin{array}{cc}
\widetilde{M}^{T} & \alpha \sigma \\
\sigma_{m+1} q^{T} & \sigma_{m+1} \alpha
\end{array}\right],
$$

where $\widetilde{M}=\widetilde{M}(q, \sigma, \gamma)$. From 19(ii) we have $\sigma_{m+1}=1 /\|\hat{q}\|=1$, and using (18) to eliminate $c_{j}$ and $s_{j}$ from (19) gives the recurrence relations stated in (20).

To obtain $\widetilde{L}$ we use Eq. (21) and the fact that $\widetilde{Q}$ is orthogonal. Thus

$$
\begin{aligned}
\widetilde{A} & =\widetilde{A} \widetilde{Q}^{T} \widetilde{Q}=\left(I_{m+1}-\hat{q} \hat{q}^{T}\right) \widetilde{Q}^{T} \widetilde{Q}=\left(\widetilde{Q}^{T}-\hat{q} e_{m+1}^{T}\right) \widetilde{Q} \\
& =\left(\left[\begin{array}{cc}
\widetilde{M} & q \\
\alpha \sigma^{T} & \alpha
\end{array}\right]-\hat{q} e_{m+1}^{T}\right) \widetilde{Q}=\left[\begin{array}{cc}
\widetilde{M} & 0 \\
\alpha \sigma^{T} & 0
\end{array}\right] \widetilde{Q} \equiv \widetilde{L} \widetilde{Q},
\end{aligned}
$$

as required. 
THeOREM A4 ( $L D V$ FACtorization OF AN ELEMENTARy MATRix). Let $A$ be $a$ matrix of the form

$$
A=D-\left[\begin{array}{c}
p \\
\alpha_{2}
\end{array}\right]\left[\begin{array}{ll}
p^{T} & \alpha_{2}
\end{array}\right]
$$

where $p$ is an $m$-vector, $\alpha_{1}$ and $\alpha_{2}$ are nonzero scalars,

$$
D=\operatorname{diag}\left(d_{1}, d_{2}, \ldots, d_{m}, \alpha_{1}^{2}\right) \equiv\left[\begin{array}{cc}
D_{1} & \\
& \\
& \alpha_{1}^{2}
\end{array}\right]
$$

and

$$
\left\|D^{-1 / 2}\left[\begin{array}{c}
p \\
\alpha_{1}
\end{array}\right]\right\|^{2}=p^{T} D_{1}^{-1} p+\frac{\alpha_{2}^{2}}{\alpha_{1}^{2}}=1
$$

The matrix $A$ has an $L D V$ factorization $A=\hat{L} \hat{D} \hat{V}$ where

$$
\hat{L}=\left[\begin{array}{cc}
\widetilde{M} & 0 \\
\alpha_{2} \beta^{T} & 0
\end{array}\right], \quad \hat{D}=\operatorname{diag}\left(\bar{d}_{1}, \bar{d}_{2}, \ldots, \bar{d}_{m}, 1\right), \quad \hat{V}=\left[\begin{array}{cc}
\widetilde{M}^{T} & \alpha_{2} \beta \\
p^{T} & \alpha_{2}
\end{array}\right],
$$

and the matrix $\hat{D}^{1 / 2} \hat{V} D^{-1 / 2}$ is orthogonal. The matrix $\widetilde{M}=\widetilde{M}(p, \beta)$ is a special lowertriangular matrix and the vectors $\bar{d}$, and $\beta$ are generated by the following recurrence relations:

$$
\left.\begin{array}{rl}
\text { 23(i) } & \text { define } t_{m+1}=\alpha_{2}^{2} / \alpha_{1}^{2} ; \\
\text { 23(ii) } & \text { for } j=m, m-1, \ldots, 1 \text { set } \\
& t_{j}=t_{j+1}+p_{j}^{2} / d_{j}, \\
& \bar{d}_{j}=d_{j} t_{j+1} / t_{j}, \\
& \beta_{j}=-p_{j} /\left(d_{j} t_{j+1}\right) .
\end{array}\right\}
$$

Proof. The matrix to be factorized can be written as

$$
A=D-\left[\begin{array}{c}
p \\
\alpha_{2}
\end{array}\right]\left[\begin{array}{ll}
p^{T} & \alpha_{2}
\end{array}\right]=D^{1 / 2}\left(I_{m+1}-\hat{q} \hat{q}^{T}\right) D^{1 / 2}=D^{1 / 2} \widetilde{A} D^{1 / 2}
$$

where

$$
q=D_{1}^{-1 / 2} p, \quad \alpha=\alpha_{2} / \alpha_{1}, \quad \hat{q}=\left[\begin{array}{l}
q \\
\alpha
\end{array}\right], \quad \widetilde{A}=I_{m+1}-\hat{q} \hat{q}^{T} .
$$

The requirement $p^{T} D_{1}^{-1} p+\alpha_{2}^{2} / \alpha_{1}^{2}=1$ ensures that $q^{T} q+\alpha^{2}=\|\hat{q}\|^{2}=1$; and hence we know from Theorem $\mathrm{A} 3$ that $\widetilde{A}$ has the orthogonal factorization $\widetilde{A}=\widetilde{L} \widetilde{Q}$, where $\widetilde{L}$ and $\widetilde{Q}$ are constructed from the quantities $q, \sigma, \gamma$ and $\alpha$ as shown. Let us define

$$
\left.\begin{array}{rl}
\delta_{j} & =d_{j}^{1 / 2} \gamma_{j}, \quad \bar{d}_{j}=\delta_{j}^{2}, \quad \beta_{j}=\sigma_{j} / \delta_{j}, \\
\Delta & =\operatorname{diag}\left(\delta_{1}, \delta_{2}, \ldots, \delta_{m}, 1\right), \quad e=(1,1, \ldots, 1)^{T} .
\end{array}\right\}
$$


Using the notation of Theorem A3 we now have

$$
A=D^{1 / 2} \widetilde{A} D^{1 / 2}=\left(D^{1 / 2} \widetilde{L}\right)\left(\widetilde{Q} D^{1 / 2}\right),
$$

where

$$
\begin{aligned}
D^{1 / 2} \widetilde{L} & =\left[\begin{array}{cc}
D_{1}^{1 / 2} & 0 \\
& \alpha_{1}
\end{array}\right]\left[\begin{array}{cc}
\tilde{M}(q, \sigma, \gamma) & 0 \\
\alpha \sigma^{T} & 0
\end{array}\right]=\left[\begin{array}{cc}
\widetilde{M}(p, \sigma, \delta) & 0 \\
\alpha_{1} \alpha \sigma^{T} & 0
\end{array}\right] \\
& =\left[\begin{array}{cc}
\widetilde{M}(p, \beta, e) & 0 \\
\alpha_{2} \beta^{T} & 0
\end{array}\right] \Delta=\hat{L} \Delta
\end{aligned}
$$

and

$$
\widetilde{Q} D^{1 / 2}=\left[\begin{array}{cc}
\tilde{M}(q, \sigma, \gamma)^{T} & \alpha \sigma \\
q^{T} & \alpha
\end{array}\right]\left[\begin{array}{cc}
D_{1}^{1 / 2} & \\
& \alpha_{1}
\end{array}\right]=\left[\begin{array}{cc}
\tilde{M}(p, \sigma, \delta)^{T} & \alpha_{1} \alpha \sigma \\
p^{T} & \alpha_{1} \alpha
\end{array}\right]
$$

$$
=\Delta\left[\begin{array}{cc}
\tilde{M}(p, \beta, e)^{T} & \alpha_{2} \beta \\
p^{T} & \alpha_{2}
\end{array}\right]=\Delta \hat{V} .
$$

Combining (26a) and (26b) gives $A=\hat{L} \hat{D} \hat{V}$ where $\hat{L}$ and $\hat{V}$ are the matrices defined in (22), and

$$
\hat{D}=\Delta^{2}=\operatorname{diag}\left(\delta_{1}^{2}, \delta_{2}^{2}, \ldots, \delta_{m}^{2}, 1\right)=\operatorname{diag}\left(\bar{d}_{1}, \bar{d}_{2}, \ldots, \bar{d}_{m}, 1\right) .
$$

Equation (26b) also gives the orthogonal matrix $\widetilde{Q}$ as

$$
\widetilde{Q}=\Delta \hat{V} D^{-1 / 2}=\hat{D}^{1 / 2} \hat{V} D^{-1 / 2}
$$

as required.

Using the definitions of $\rho_{j}, \sigma_{j}, \gamma_{j}$ and $q_{j}$ in (20) and (24), we can now simplify the expressions for $\bar{d}_{j}$ and $\beta_{j}$ in (25) as follows:

$$
\begin{aligned}
& \bar{d}_{j}=d_{j} \gamma_{j}^{2}=d_{j} \rho_{j+1}^{2} / \rho_{j}^{2} \\
& \beta_{j}=\sigma_{j} /\left(\gamma_{j} d_{j}^{1 / 2}\right)=-q_{j} /\left(\rho_{j+1}^{2} d_{j}^{1 / 2}\right)=-p_{j} /\left(\rho_{j+1}^{2} d_{j}\right) .
\end{aligned}
$$

From 20(i) we also have $\rho_{m+1}^{2}=\alpha^{2}=\alpha_{2}^{2} / \alpha_{1}^{2}$. Finally, as in Theorem A2, we define $t_{j}=\rho_{j}^{2}$ to avoid the computation of square roots. The recurrence relations (23) now follow and the theorem is proved.

LemMA A3 (CHOLESKY FACTORS OF $D_{1}+p p^{T}$ ). If $p$ is an m-vector and $D_{1}=$ $\operatorname{diag}\left(d_{1}, d_{2}, \ldots, d_{m}\right)$ where $d_{i}>0$, the Cholesky factorization of $D_{1}+p p^{T}$ is

$$
D_{1}+p p^{T}=\widetilde{M} D_{2} \widetilde{M}^{T}
$$

where

$$
D_{2}=\operatorname{diag}\left(\bar{d}_{1}, \bar{d}_{2}, \ldots, \bar{d}_{m}\right), \quad \widetilde{M}=\widetilde{M}(p, \beta)
$$

with 


$$
\begin{aligned}
\widetilde{M} D_{2} \beta & =p, \\
\beta^{T} D_{2} \beta & =1-\alpha^{2}>0 .
\end{aligned}
$$

The quantities $\bar{d}_{j}, \beta_{j}$ and $\alpha^{2}$ are given by the recurrence relations (12).

Proof. Using the notation and results of Theorem A2, we can write down the $L D V$ factorization

$$
\left[\begin{array}{ll}
D_{1} & p \\
& \\
& 1
\end{array}\right]=\hat{L} \hat{D} \hat{V}
$$

If

$$
D=\left[\begin{array}{ll}
D_{1} & \\
& \\
& 1
\end{array}\right], \quad \hat{D}=\left[\begin{array}{ll}
D_{2} & \\
& \alpha^{2}
\end{array}\right], \quad \widetilde{Q}=\hat{D}^{1 / 2} \hat{V} D^{-1 / 2},
$$

then the matrix $\widetilde{Q}$ is orthogonal. Post-multiplying (28) by $D^{-1 / 2}$ gives

$$
\left[\begin{array}{ll}
D_{1}^{1 / 2} & p \\
& 1
\end{array}\right]=\hat{L} \hat{D} \hat{V} D^{-1 / 2}=\hat{L} \hat{D}^{1 / 2} \widetilde{Q}
$$

and since $\widetilde{Q} \widetilde{Q}^{T}=I$ we have

$$
\left[\begin{array}{ll}
D_{1}^{1 / 2} & p \\
& 1
\end{array}\right]\left[\begin{array}{cc}
D_{1}^{1 / 2} & \\
p^{T} & 1
\end{array}\right]=\hat{L} \hat{D} \hat{L}^{T}=\left[\begin{array}{ll}
\widetilde{M} & \\
\beta & 1
\end{array}\right]\left[\begin{array}{ll}
D_{2} & \\
& \alpha^{2}
\end{array}\right]\left[\begin{array}{ll}
\widetilde{M}^{T} & \beta \\
& 1
\end{array}\right] .
$$

Hence

$$
\left[\begin{array}{cc}
D_{1}+p p^{T} & p \\
p^{T} & 1
\end{array}\right]=\left[\begin{array}{cc}
\widetilde{M} D_{2} \widetilde{M}^{T} & \widetilde{M} D_{2} \beta \\
\beta^{T} D_{2} \widetilde{M}^{T} & \beta^{T} D_{2} \beta+\alpha^{2}
\end{array}\right]
$$

and relations (27) follow immediately.

Lemma A4 (ChOlesky factors of $D_{1}-p p^{T}$ ). If $p$ is an m-vector, $D_{1}=$ $\operatorname{diag}\left(d_{1}, d_{2}, \ldots, d_{m}\right)$ where $d_{i}>0$ and $\alpha^{2}=1-p^{T} D_{1}^{-1} p>0$, the Cholesky factorization of $D_{1}-p p^{T}$ is

$$
D_{1}-p p^{T}=\tilde{M} \widetilde{D}_{2} \widetilde{M}^{T}
$$

where

$$
D_{2}=\operatorname{diag}\left(\bar{d}_{1}, \bar{d}_{2}, \ldots, \bar{d}_{m}\right), \quad \widetilde{M}=\widetilde{M}(p, \beta)
$$

with

$$
\begin{aligned}
& \tilde{M} D_{2} \beta=-p, \\
& \beta^{T} D_{2} \beta=1 / \alpha^{2}-1>0 .
\end{aligned}
$$

The quantities $\bar{d}_{j}$ and $\beta_{j}$ are defined by the recurrence relations (23), with 23(i) replaced by $t_{m+1}=\alpha^{2}$.

Proof. Using the notation and results of Theorem A4, we have $\alpha^{2}=\alpha_{2}^{2} / \alpha_{1}^{2}$ and the $L D V$ factorization 
$\left[\begin{array}{ll}D_{1} & \\ & \alpha_{1}^{2}\end{array}\right]-\left[\begin{array}{c}p \\ \alpha_{2}\end{array}\right]\left[\begin{array}{ll}p^{T} & \alpha_{2}\end{array}\right]=\hat{L} \hat{D} \hat{V}\left(=\hat{L} \hat{D} \hat{L}^{T}\right)=\left[\begin{array}{cc}\widetilde{M} & \\ \alpha_{2} \beta^{T} & 0\end{array}\right]\left[\begin{array}{ll}D_{2} & \\ & 1\end{array}\right]\left[\begin{array}{cc}\widetilde{M}^{T} & \alpha_{2} \beta \\ p^{T} & \alpha_{2}\end{array}\right]$.

Hence

$$
\left[\begin{array}{cc}
D_{1}-p p^{T} & -\alpha_{2} p \\
-\alpha_{2} p^{T} & \alpha_{1}^{2}-\alpha_{2}^{2}
\end{array}\right]=\left[\begin{array}{cc}
\tilde{M} D_{2} \widetilde{M}^{T} & \alpha_{2} \widetilde{M} D_{2} \beta \\
\alpha_{2} \beta^{T} D_{2} \widetilde{M}^{T} & \alpha_{2}^{2} \beta^{T} D_{2} \beta
\end{array}\right]
$$

and relations (29) follow immediately.

Division of Numerical Analysis and Computing

National Physical Laboratory

Teddington, Middlesex, England

Division of Numerical Analysis and Computing

National Physical Laboratory

Teddington, Middlesex, England

Applied Mathematics Division

DSIR, Wellington, New Zealand

1. R. FLETCHER \& M. J. D. POWELL (1973), On the Modification of $L D L^{T}$ Factorizations, AERE report, TP 519.

2. W. M. GENTLEMAN (1973), "Least squares computations by Givens transformations without square roots," J. Inst. Math. Appl., v. 12, pp. 329-336. MR 48 \#7575.

3. P. E. GILL, G. H. GOLUB, W. MURRAY \& M. A. SAUNDERS (1974), "Methods for modifying matrix factorizations," Math. Comp., v. 28, pp. 505-535.

4. E. HELLERMAN \& D. RARICK (1971), "Reinversion with the preassigned pivot procedure," Math. Programming, v. 1, pp. 195-216. MR 45 \#2895

5. E. HELLERMAN \& D. RARICK (1972), "The partitioned preassigned pivot procedure $\left(\mathrm{P}^{4}\right), "$ Sparse Matrices and Their Applications, D. J. Rose and R. A. Willoughby (Editors), Plenum Press, New York, pp. 67-76. MR 48 \#10075.

6. M. A. SAUNDERS (1972), Product Form of the Cholesky Factorization for Large-Scale Linear Programming, Report STAN-CS-72-301, Computer Science Department, Stanford University, Stanford, California. 